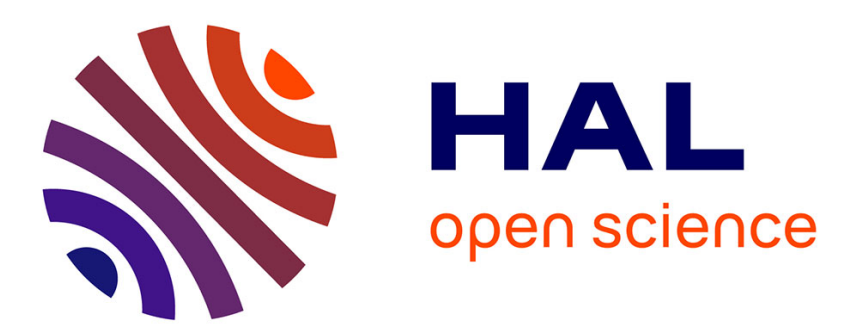

\title{
Reduced Locomotion Dynamics with Passive Internal DoFs: Application to Non-holonomic and Soft Robotics
}

\author{
Frédéric Boyer, Ayman Belkhiri
}

\section{To cite this version:}

Frédéric Boyer, Ayman Belkhiri. Reduced Locomotion Dynamics with Passive Internal DoFs: Application to Non-holonomic and Soft Robotics. IEEE Transactions on Robotics, 2013, pp.1-16. hal00842093

\section{HAL Id: hal-00842093 \\ https://hal.science/hal-00842093}

Submitted on 22 Sep 2013

HAL is a multi-disciplinary open access archive for the deposit and dissemination of scientific research documents, whether they are published or not. The documents may come from teaching and research institutions in France or abroad, or from public or private research centers.
L'archive ouverte pluridisciplinaire HAL, est destinée au dépôt et à la diffusion de documents scientifiques de niveau recherche, publiés ou non, émanant des établissements d'enseignement et de recherche français ou étrangers, des laboratoires publics ou privés. 


\title{
Reduced locomotion dynamics with passive internal DoFs: Application to non-holonomic and soft robotics
}

\author{
Frédéric Boyer, Ayman Belkhiri
}

\begin{abstract}
This article proposes a general modelling approach for locomotion dynamics of Mobile Multibody Systems (MMS) containing passive internal degrees of freedom (dofs) concentrated into (ideal or not) joints and/or distributed along deformable bodies of the system. The approach embraces the case of non-holonomic mobile multibody systems with passive wheels, the pendular climbers and the locomotion systems bio-inspired by animals that exploit the advantages of soft appendages such as the fish swimming with their caudal fin or the moths using the softness of their flapping wings to improve flight performance. The article proposes a general structured modelling approach of MMS with tree-like structures along with efficient computational algorithms of the resulting equations. The approach is illustrated through non-trivial examples such as the 3D bicycle and a compliant version of the snake-board.
\end{abstract}

Index Terms-Locomotion dynamics, biologically inspired robots, soft robotics, non-holonomic systems.

\section{INTRODUCTION}

$\mathbf{N}$ ATURAL or artificial, any locomotion system undergoes two types of motion. The first are overall rigid motions of degrees of freedom (dofs) called "external dofs" because they are measured by an observer outside the system. Since these dofs are not directly actuated, their time evolution is governed by a model of locomotion (i.e. the contact between the system and the world). The second type includes the motions of the internal dofs or shape dofs [1]-[3]. Among these internal dofs, some are "unactuated" or "passive" and play a key role in the locomotion of animals and robots [4], [5]. For instance, they can help to simplify the control laws or to increase the number of dofs used in locomotion without increasing the number of actuators. They also allow one to save consumed energy, to extract it from the environment and to store it in some of the internal dofs before restoring it in the external dofs. They can also be used to increase the instantaneous power beyond the intrinsic capabilities of actuators [6]. As illustrations from natural world, let us mention the climbing animals which use pendular locomotion or those which use compliant appendages to generate thrust or lift as in swimming of fish or hovering flight of moths [7]. In the first case, the animal does not seek to resist the gravity field as in static locomotion, but on the contrary, uses its potential energy as a resource of motivity by swinging up by the supports [8]. Inspired by gibbons' brachiation, this strategy is exploited by

F. Boyer and A. Belkhiri are with the Institut de Recherche en Communication et Cybernétique de Nantes (IRCCyN) and Ecole des Mines de Nantes, Nantes, France.

Manuscript received April 3, 2013; revised Septembre 6, 2013. the most efficient climbing artifacts [9], [10]. In the case of swimming, experiments and modelling works have recently shown that a compliant dead fish can resonate in a Karman vortex street and extract from the ambient vorticity the energy required to resist drag forces and maintain its position in the flow [11], [12]. Similarly, the hovering flight of moths involves a (passive) wing twisting deformation which is the key of lift generation. Beyond these natural examples, many artificial wheeled systems use passive wheels to self-propel (snakelike robots, snake-board) [13] or to self-stabilize (the bicycle) [14]. Known today as dynamic non-holonomic systems, these systems differ from kinematic non-holonomic systems by the fact that the time-evolution of their external dofs requires dynamics to be modelled [1], [15].

To date and so far, there is no unified modelling framework able to embrace all these systems and in particular those which contain bodies with distributed compliancies. Therefore, the purpose of this article is to contribute to such a framework. In order to achieve this objective, we consider any robot (or any animal in a bio-mimetic approach) as a Mobile Multibody System (MMS), i.e. a set of solid bodies connected by internal joints whose time-variations produce (by reaction) external forces that generate the expected "net displacements". Following geometric mechanics [2], [13], [16], [17], the model of these systems is derived by applying the laws of dynamics on a space of configurations $(g, r)$ where $g$ is the transformation of a Lie group $G$ which parameterizes the net motion of the MMS, while $r$ parameterizes the shape of the MMS and coordinatizes a manifold $\mathcal{S}$, named the "shape space" of the MMS. In the rest of the article, we consider MMS that are possibly subject to external forces depending on the current position, velocities and accelerations of the system and/or to eventual kinematic (external) constraints which can, for instance, model the contacts of the system with a rigid substrate. In the constrained case, an outstanding study revealing the geometric insights of non-holonomic systems has been proposed in [18]. However, this general approach is restricted to rigid fully actuated MMS. By contrast, we here consider the case of MMS with internal unactuated (passive) degrees of freedom, some of them accounting for eventual flexibilities distributed along the bodies. As such, the article here presented, proposes an extension of the context of [18] to the case of soft MMS. Another novelty of the article concerns the dynamic model of the internal actuated dofs, which is studied not only in its forward form but also in its inverse form. All, these extensions raise new difficulties. In particular, they need to tackle in a 
unified formalism systems whose passive dofs can be ruled by kinematic constraints (as the angle of the front wheel of a bicycle), or actual dynamic equations (as the deformations of a flapping wing or those of the elastic rotor of a soft snakeboard). Moreover, addressing the dynamics of the internal actuated dofs in the inverse form, needs to tackle redundant degrees of freedom in torque inputs which do not produce any passive or active motion and simply serve to cancel each other as is the case for a planar mobile robot with two redundant wheels operating in opposition. As a result, this other new aspect can help roboticists to address locomotion problems where the control objectives are not only related to the net motions but also to hyperstatic forces as those involved in the stability of redundant legged locomotion or hyper-redundant snakes.

In order to achieve these extensions, the dynamics of the passive (internal and external) velocities (more simply named "passive dynamics") are projected onto the kernel of the kinematic constraints locked in the current configuration of the actuated dofs. This choice provides a systematic modelling approach structured in a kinematic and a dynamic stage. As regards the compliances, they can be localised on passive joints or distributed along the bodies. In this latter case, the body deformations are modelled by using the floating frame approach [19] and the assumed modes method [20] as initially developed in the context of flexible multibody systems dynamics [21], [22], [23]. This choice is motivated by the dramatic reduction of resulting equations, a useful characteristic for roboticists working on feedback control, optimization, etc. In addition to offering a modelling framework to a wide range of systems, the article also proposes some efficient algorithmic tools for the practical calculation of the models of these systems. In particular, the kinematic modeling is investigated under the generic point of view of generalized inversion while the dynamics of a MMS possibly containing compliant bodies is computed thanks to an extension of the Luh algorithm [24], proposed in [23] and here used in a way which to our knowledge is new in itself. For the sake of illustration, the approach is finally applied to several non-trivial examples such as the 3D bicycle and an elastic (soft) version of the snake-board.

The article is structured as follows. In section II, we consider the kinematic model of a MMS subject to an arbitrary number of non-holonomic constraints, then we introduce the problem we will address in the rest of the article. By a projection process of the MMS dynamics onto the kernel of the constraints, we derive in section III the reduced forward dynamics of the passive (internal and external) dofs controlled by the internal actuated dofs. Once these dynamics are known, it becomes possible to reconstruct the motion of all the dofs (internal and external, actuated and passive) and to compute the internal torques which have to be applied onto the actuated joints by the actuators to ensure these motions. It is the subject of section IV to derive this model which is nothing else than the inverse internal dynamics of the MMS. When the number of bodies exceeds a few ones, it becomes too much difficult to calculate its dynamic model by hand. Therefore, we address

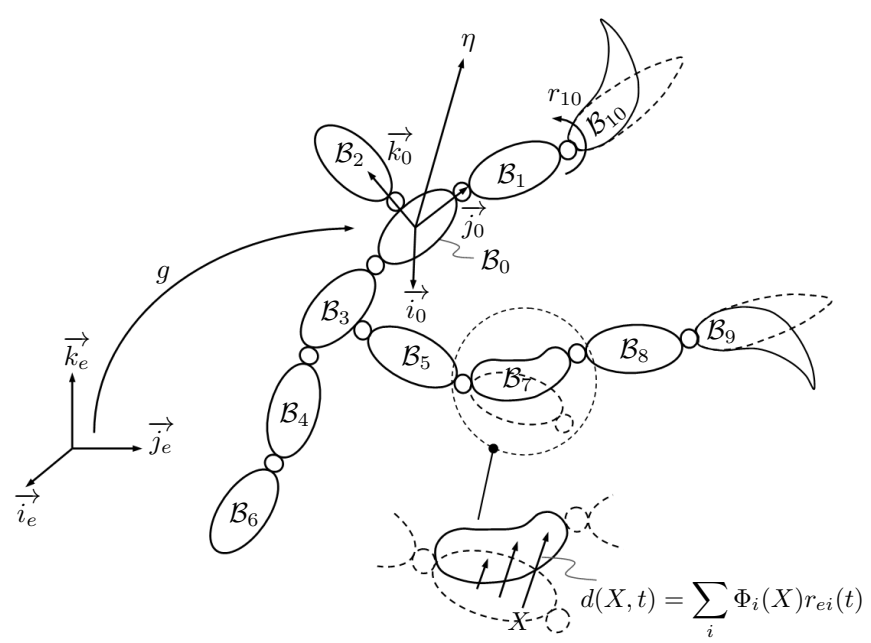

Fig. 1. Mechanical structure of a tree-like MMS: The bodies are labelled in increasing order from the reference body to the tips. In this case, $\mathcal{B}_{9}$ and $\mathcal{B}_{10}$ are two terminal compliant organs while $\mathcal{B}_{7}$ is an intermediate compliant body.

the problem of its efficient calculation in section V. Sections VI and VII are devoted to the application of the approach on illustrative examples. At last, section VIII closes the article with some concluding remarks.

\section{Preamble}

The purpose of this preamble is twofold. First, it introduces the context of the following results, in particular the assumptions, conventions and basic notations on which they are based. Second, it presents the final set of dynamic equations.

\section{A. Basic framework}

In the subsequent developments, we will consider a tree-like MMS as that pictured in Fig. 1. Such a system is composed of $N$ solid bodies possibly compliant, and connected by onedof joints that for the sake of simplicity we will assume to be of angular nature. The deformation of each compliant body is measured with respect to a mobile rigid configuration defined at each instant as the extension of the resting geometry of the body from the joint which precedes it in the chain ${ }^{1}$ (drawn in dotted line on Fig. 1). According to a Rayleigh-Ritz approach [20], these deformation fields are then reduced on a truncated functional basis of assumed modes as for instance that of the first natural modes of the cantilevered bodies. With such a finite parametrisation of the bodies' deformation, any configuration of the MMS is naturally defined by a pair $(g, r) \in G \times \mathcal{S}$ where $G$ is the group of the net displacements (namely a subgroup of $S E(3)$ or $S E(3)$ itself) of a reference frame attached to an arbitrarily distinguished body, named reference body, and $\mathcal{S}$ is the shape space, parameterized by the joint and Rayleigh-Ritz coordinates gathered in the vector $r$, which defines the "internal dofs". The active and passive

\footnotetext{
${ }^{1}$ Such a reference configuration defines at each instant a frame which floats around the real body. For this reason, the approach was once known as the "floating frame approach" [19].
} 
internal dofs are distinguished by splitting the shape space $\mathcal{S}$ into $\mathcal{S}=\mathcal{S}_{p} \times \mathcal{S}_{a}$, with $\mathcal{S}_{p}$ the space of the passive shape dofs and $\mathcal{S}_{a}$ that of the active ones. The dimension of the fiber is noted $n$, that of $\mathcal{S}$ is $s$ while $s_{p}$ and $s_{a}$ are those of $\mathcal{S}_{p}$ and $\mathcal{S}_{a}$ with $s=s_{p}+s_{a}$ and $r=\left(r_{p}^{T}, r_{a}^{T}\right)^{T}$ is the "passive-active" block partition of $r$. The influence of the environment is modelled through external forces generally depending on the current state of the system and/or a set of $m$ independent persistent and non-holonomic kinematic constraints. In these conditions, a first formulation of the entire (external and internal) dynamics is given by applying the principles of Lagrangian dynamics on the configurations space $G \times \mathcal{S}_{p} \times \mathcal{S}_{a}$, which leads to:

$$
\begin{aligned}
\left(\begin{array}{lll}
\mathcal{M} & M_{p}^{T} & M_{a}^{T} \\
M_{p} & m_{p p} & m_{p a} \\
M_{a} & m_{a p} & m_{a a}
\end{array}\right)\left(\begin{array}{c}
\dot{\eta} \\
\ddot{r}_{p} \\
\ddot{r}_{a}
\end{array}\right)= \\
\left(\begin{array}{c}
f \\
Q_{p} \\
Q_{a}+\tau_{a}
\end{array}\right)+\left(\begin{array}{c}
A^{T} \\
B_{p}^{T} \\
B_{a}^{T}
\end{array}\right) \lambda,
\end{aligned}
$$

where from left to right, we find: the $r$-dependent inertia matrix of the system. The vector of accelerations on $G \times \mathcal{S}_{a} \times \mathcal{S}_{p}$ with $\eta=g^{-1} \dot{g}$ an element of the Lie algebra $\mathfrak{g}$ of $G$ which defines the velocity of the reference frame in its mobile axis. The vector of inertial, external and internal forces including the torques provided by the actuators $\tau_{a}$ along with the friction and restoring forces introduced by the compliances concentrated on some of the passive joints or distributed along the compliant bodies $^{2}$. Finally, the last term (on the right) represents the vector of generalized forces imposed by the constraints, with $\lambda$ a set of Lagrange multipliers forcing the constraints. With our definition of the configuration space, these constraints can be written under the general form:

$$
A \eta+B_{p} \dot{r}_{p}+B_{a} \dot{r}_{a}=0,
$$

with $A, B_{p}$ and $B_{a}$ being $m \times n, m \times s_{p}$ and $m \times s_{a}$ matrices which only depend on $r$. Exploiting these constraints, we will see that the time evolution of some of the passive coordinates can be deduced directly from the actuated ones through kinematics, while others will require invoking dynamics. The first type of coordinates are named "passive kinematic coordinates" and denoted $r_{p \text {, kin }}$ while the others are named "passive dynamic coordinates" and noted $r_{p, d y n}$. We will distinguish the "unlocked" constraints (2) which relate together the components of $\left(\eta, \dot{r}_{p}, \dot{r}_{a}\right)$ from their "locked" counterpart which are given by:

$$
A \eta+B_{p} \dot{r}_{p}=0
$$

i.e. by imposing $\dot{r}_{a}=0$ in (2). Physically, the unlocked constraints (2) represent the kinematic conditions imposed

\footnotetext{
${ }^{2}$ Going further into the details, the forces exerted on the external and internal actuated dofs can be decomposed in inertial (Coriolis and centrifugal forces) and external ones (excluding those imposed by the constraints) as follows: $f=f_{\text {inert }}(r, \dot{r}, \eta)+f_{\text {ext }}, Q_{a}=Q_{a, \text { inert }}(r, \dot{r}, \eta)+Q_{a, \text { ext }}$ while $Q_{p}=Q_{p, \text { inert }}+Q_{p, \text { ext }}+Q_{p, \text { int }}$ contains a third component $Q_{p, \text { int }}\left(r_{p}, \dot{r}_{p}\right)$ modelling the passive restoring and friction internal forces. As regards the external forces, in the general case, they depend on the entire state $(g, r, \eta, \dot{r})$.
}

by the contacts when the actuated joints are free to move according to their imposed time evolution (which is assumed to be compatible with the mobility of the MMS), while (3) represents the same constraints but when the actuated joints $r_{a}$ are locked in their current position. Based on this distinction, we denote by $m^{o}\left(m^{o} \leq m\right)$ the total number of independent locked constraints, and by $m$, that of unlocked constraints.

\section{B. Statement of a locomotion problem}

To derive the expected model, we address the following fundamental locomotion problem [15]. Considering the actuated joint variables of $r_{a}$ as imposed control inputs given by their time evolution $t \mapsto r_{a}(t)$, solve the forward passive (external and internal) dynamics, i.e. compute at each step of time $t$ of an integration time-loop: the passive accelerations $\left(\ddot{g}, \ddot{r}_{p}\right)$ from the knowledge of the current state $\left(g, r_{p}, \dot{g}, \dot{r}_{p}\right)$ and that of the current inputs $\left(r_{a}, \dot{r}_{a}, \ddot{r}_{a}\right)(t)$. This first subproblem corresponds to solve the forward passive dynamics controlled by the actuated dofs. Once $\left(\ddot{g}, \ddot{r}_{p}\right)$ computed, a second subproblem consists in calculating the current value of the joint torques $\tau_{a}$ required by the motions of all the (passive and actuated) dofs, i.e. to derive and solve the inverse internal actuated dynamics. Note here that, more than leading to the expected model, this problem has also a strong practical interest in robotics and control, since the locomotion gaits of a robot are primarily specified in terms of shape motions, while in a second step the torques are computed to check the feasibility of the shape motion control. Finally, when addressing this fundamental problem, in all the subsequent developments, the time dependency of actuated variables is explicitly indicated (i.e. $r_{a}=r_{a}(t)$ ) while all the other (passive) variables $r_{p}$ and $g$ depend on the time only implicitly, i.e. through the dynamics that we are seeking for. At last, we will see that the forward dynamics of actuated dofs can be easily deduced from its inverse version.

\section{Introduction to the final set of equations}

Before pursuing, let us comment a little further on (1-2). In fact this first formulation cannot be deduced from Lagrange equations but rather from Poincaré equations [25], [26] which extend Lagrange equations to systems whose configuration space is defined as a non commutative Lie group (in our case $G \times \mathbb{R}^{s}$ ). Compared to any formulation derived from Lagrange equations, the advantage of the Poincare formulation lies in the fact that in (1-2), the dependence of $g$ is confined to the vector of external forces through gravity or any force which breaks the symmetry of the ambient space. As a result, (1-2) is more simple than any formulation derived from Lagrange equations where the net motions are parameterized on $\mathbb{R}^{n}$ using Euler angles for instance. In the language of geometric mechanics, when it does not contain any $g$-dependent external forces, the intrinsic formulation (1-2) can be interpreted as the result of a first (preliminary) reduction (from $(g, \dot{g})$ to $\eta$ ) of the system dynamics. In the following, we will deal with a second reduction process which aims at removing reaction unknowns $\lambda$ (and their associated constraints) in (1-2). At the 
TABLE I

VALUES OF $H$ AND $\lambda_{\text {stat }}$ IN (4-8).

\begin{tabular}{|c|c|c|}
\hline & $m=m^{o}$ & $m>m^{o}$ \\
\hline$n+s_{p}=m^{o}$ & $\lambda_{\text {stat }}=0, H=0$ & $\lambda_{\text {stat }} \neq 0, H=0$ \\
\hline$n+s_{p}>m^{o}$ & $\lambda_{\text {stat }}=0, H \neq 0$ & $\lambda_{\text {stat }} \neq 0, H \neq 0$ \\
\hline
\end{tabular}

end of this second reduction process, we will see that the timeevolution of a MMS which contains passive internal degrees of freedom (including those induced by distributed compliances) and actuated internal dofs, can be stated as follows:

$$
\begin{aligned}
& \dot{\eta}_{r}=\widetilde{\mathcal{M}}_{r}^{-1} \widetilde{\mathcal{F}}_{r}, \\
& \ddot{r}_{p, d y n}=m_{p p, r}^{-1} \widetilde{Q}_{p, r}, \\
& \tau_{a}=\widetilde{m}_{a a} \ddot{r}_{a}(t)-\widetilde{Q}_{a}+B_{a}^{T} \lambda_{s t a t}, \\
& \dot{g}=g\left(J_{e x t} \dot{r}_{a}(t)+H_{e e} \eta_{r}+H_{e p} \dot{r}_{p, d y n}\right), \\
& \dot{r}_{p}=J_{i n t} \dot{r}_{a}(t)+D \dot{r}_{p, d y n} .
\end{aligned}
$$

From top to bottom, we find the forward dynamics of the net reduced velocities, the forward dynamics of the passive dynamic dofs, the inverse dynamics of the actuated dofs, the reconstruction equation of the net motions and the reconstruction equation of the passive internal shape motions. Without entering too much into the details of the expressions, we introduced the following notations. The index $r$ means "reduced", while a tilde over a matrix denotes a "modified" version of this matrix in a certain sense we will explain later and which is such that when there are no passive dofs, the modified matrices coincide with the original ones. $\widetilde{\mathcal{M}}_{r}(r)$ and $\widetilde{\mathcal{F}}_{r}\left(g, r, \dot{r}, \ddot{r}_{a}(t), \eta\right)$ represent the inertia matrix and the vector of external and inertial generalized forces of the reduced external dynamics (of net motions). $m_{p p, r}(r)$ and $\widetilde{Q}_{p, r}\left(g, r, \dot{r}, \ddot{r}_{a}(t), \eta\right)$ are the inertia matrix and the vector of internal, external and inertial generalized forces of the passive dynamic dofs. $\widetilde{m}_{a a}(r)$ and $\widetilde{Q}_{a}\left(g, r, \dot{r}, \ddot{r}_{a}(t), \eta\right)$ are the inertia matrix and the vector of generalized external and inertial forces applied onto the active internal dofs, while $B_{a}^{T} \lambda_{\text {stat }}$ is the vector of the generalized forces (reaction torques) possibly exerted by the constraints when they are redundant i.e. hyperstatic. Finally, all the reminding matrices can be deduced from (2). In particular, $J_{\text {ext }}, J_{\text {int }}$ represent some Jacobian matrices while the columns of $H_{e e}, H_{e p}$ and $D$ are sub-blocks of $H$, a matrix whose columns span the kernel of the locked constraints. Equations (4-8) define the general formulation of an arborescent MMS containing internal localized or distributed passive dofs, subject to external forces and non-holonomic constraints. Furthermore, we will see that depending on the relative values of $m$ and $m^{o}$, these equations can be specified as indicated in Table I. Before closing this section, let us make a further remark.

Remark 1: Because (4-8) is the solution of the locomotion problem of section II-B, the dynamics of internal actuated dofs appear in their inverse form (6), where $r_{a}$ is imposed by its time-evolution, and $\lambda_{\text {stat }}$ represents contact reaction forces which do not produce any acceleration of the passive dofs $^{3}$. Alternatively, the forward formulation of the dynamics of the MMS controlled by the vector of torques $\tau_{a}=\tau_{a}(t)$ can be easily deduced from equations (4-8) by replacing in them, $r_{a}(t)$ by $r_{a}$, while the inverse dynamics (6) have to be replaced by:

$$
\ddot{r}_{a}=\widetilde{m}_{a a}^{-1}\left(\tau_{a}(t)+\widetilde{Q}_{a}\right),
$$

where $\lambda_{\text {stat }}$ does not appear anymore in the forward case $\mathrm{c}^{4}$.

\section{FORWARD DYNAMICS OF THE PASSIVE DOFS}

According to the objectives of section II-B, we derive in this section a model allowing to reconstruct the motion of passive (internal and external) dofs $r_{p}$ and $g$ from the motions imposed to the internal actuated dofs $r_{a}$. To derive such a model, the idea consists in trying to express the maximum of passive dofs as a function of the actuated ones with the help of the kinematic constraints. In doing so, we will make appear a residual set of coordinates requiring a further dynamic model. To that end, we start from the dynamics (1-2) and consider kinematics further.

\section{A. Reduced kinematics of the passive dofs}

Since $r_{a}$ is known through its time evolution $t \mapsto r_{a}(t)$, the implicit linear algebraic system (2), can be rewritten alternatively as:

$$
A^{\ddagger} \eta^{\ddagger}+B_{a} \dot{r}_{a}(t)=0,
$$

with $A^{\ddagger}=\left(A, B_{p}\right)$ a $m \times\left(n+s_{p}\right)$ matrix, and $\eta^{\ddagger}=\left(\eta^{T}, \dot{r}_{p}^{T}\right)^{T}$, the $\left(n+s_{p}\right) \times 1$ vector of the passive (external and internal) velocities. By generalized inversion of (10), we deduce the general kinematic model under the following form:

$$
\eta^{\ddagger}=J \dot{r}_{a}(t)+H \eta_{r}^{\ddagger},
$$

where $J$ can be detailed as (the upper script $(-1)$ indicates a generalized inversion):

$$
J=-\left(A^{\ddagger}\right)^{(-1)} B_{a},
$$

while the second term of (11) represents the general form of terms living in the kernel of $A^{\ddagger}$ noted $\mathcal{K}\left(A^{\ddagger}\right)$, which is nothing but the kernel of the locked constraints (3). Going further, the columns of $H$ span a basis of $\mathcal{K}\left(A^{\ddagger}\right)$ and $\eta_{r}^{\ddagger}$ stands for a vector of reduced (passive) velocities. Now block-partitioning (11), allows one to detail the general form of the kinematic model as:

$$
\left(\begin{array}{c}
\eta \\
\dot{r}_{p}
\end{array}\right)=\left(\begin{array}{c}
J_{e x t} \\
J_{\text {int }}
\end{array}\right) \dot{r}_{a}(t)+\left(\begin{array}{cc}
H_{e e} & H_{e p} \\
H_{p e} & H_{p p}
\end{array}\right)\left(\begin{array}{c}
\eta_{r} \\
\dot{r}_{p, r}
\end{array}\right),
$$

\footnotetext{
${ }^{3}$ These forces appear in redundant locomotion systems as in the case of over-actuated snake-like robots for instance.

${ }^{4}$ This forward formulation of the dynamics is in fact related to the formulation proposed by the geometric locomotion theory of rigid fullyactuated MMS. In fact, in the case where the external forces of (1) are $g$-independent, $g$ can be removed from $\widetilde{\mathcal{F}}_{r}, \widetilde{Q}_{p, r}$ and $\widetilde{Q}_{a, r}$. Furthermore, if the internal dofs are all actuated, one can force $r_{a}=r$ and $\tau_{a}(t)=\tau(t)$ in (4), (7) and (9) while (5) and (8) are simply removed. In these restricted conditions, replacing the reduced twist $\eta_{r}$ by its conjugate momentum named non-holonomic momentum and denoted $p_{r}$, allows to rewrite the forward dynamics $(4,5,9,7,8)$ in the form of [1], [18]
} 
where $\eta_{r}$ and $\dot{r}_{p, r}$ respectively denote the (possible) reduced external and internal velocities. This last relation will be used in the following as a reduction process applied to the passive dynamics in $G \times \mathcal{S}_{p}$. In this context, (13) will be seen as a relation allowing to change the parametrization of motions from the set of passive velocities $\eta^{\ddagger}=\left(\eta^{T}, \dot{r}_{p}^{T}\right)^{T}$ to the set of reduced ones $\eta_{r}^{\ddagger}=\left(\eta_{r}^{T}, \dot{r}_{p, r}^{T}\right)^{T}$ (with of course $\operatorname{dim}\left(\eta_{r}^{\ddagger}\right) \leq$ $\left.\operatorname{dim}\left(\eta^{\ddagger}\right)=n+s_{p}\right)$. The unreduced velocities $\eta^{\ddagger}$ belong to the tangent space to $G \times \mathcal{S}_{p}$, while the reduced ones $H \eta_{r}^{\ddagger}$ live in the constrained subspace of the tangent space to $G \times \mathcal{S}_{p}$. As such, these velocities are said to be compatible with the constraints, and the space of the compatible velocities defined in each point of $G \times \mathcal{S}_{p}$ is the admissible space of velocities.

\section{B. Remarks}

Remark 2: Invoking the definition of $\eta=g^{-1} \dot{g}$, along with (13), shows that if we can compute $\eta_{r}^{\ddagger}$, then we can completely reconstruct the motion of the system using the following reconstruction equation:

$$
\frac{d}{d t}\left(\begin{array}{c}
g \\
r_{p}
\end{array}\right)=\left(\begin{array}{c}
g\left(J_{e x t} \dot{r}_{a}(t)+H_{e e} \eta_{r}+H_{e p} \dot{r}_{p, r}\right) \\
J_{\text {int }} \dot{r}_{a}(t)+H_{p e} \eta_{r}+H_{p p} \dot{r}_{p, r}
\end{array}\right),
$$

whose first (top) row can be numerically time-integrated with an intrinsic geometric integrator on $G$ [27], or alternatively with a quaternion-based integrator, while the second row only requires standard schemas on linear spaces. In the remainder of this section, we will show how $\eta_{r}^{\ddagger}$ can be computed.

Remark 3: Using the notations introduced in section II-A, the inversion of (11) shows that the model of the time-evolution of the passive dofs requires addressing two cases depending on the relative values of $m^{o}=\operatorname{rank}\left(A, B_{p}\right)=\operatorname{rank}\left(A^{\ddagger}\right)$ and $n+s_{p}=\operatorname{dim}\left(\eta^{\ddagger}\right)$ (see Table I): 1) If $m^{o}=n+s_{p}$ (this corresponds to the fully- or over-constrained case), then $H=0$ and the model of passive dofs is purely kinematic. In this case, we have two subcases depending on the relative values of $m$ and $n+s_{p}$ : 1.1) $m>n+s_{p}$ (over-constrained case) then $J=-A^{\ddagger(-1)} B_{a}$, where the generalized inverse can be deduced by inversion of $m^{o}$ independent rows of (10), while the others play the role of compatibility equations that the actuated dofs have to satisfy to preserve mobility. 1.2) $m=n+s_{p}$ (fully-constrained case), in this case $J=-A^{\ddagger-1} B_{a}$. 2) If $m^{o}<n+s_{p}$ (under-constrained case), the constraints are not sufficient to define univocally the passive (external and internal) velocities from the actuated ones. This results in the existence of a non-zero kernel $(H \neq 0)$ of the constraints in which the passive velocities are ruled by a dynamic model. In this case, once again $J=-A^{\ddagger(-1)} B_{a}$, while the elements in the kernel of $A^{\ddagger}$ represent the (external and/or internal) passive velocities allowed by the constraints when they are locked in the current values of $r_{a}$.

Remark 4: In (13), $\dot{r}_{p, r}$ represents a vector of reduced internal passive velocities modelling eventual holonomic constraints relating the internal passive dofs. More generally, these constraints could mix the external and internal dofs in such a manner that the "external-internal" partition is not justified anymore. Finally, $\left(\eta_{r}^{T}, \dot{r}_{p, r}^{T}\right)^{T}$ could be more generally replaced by a vector of reduced non-integrable velocities $\nu_{r p}$.

Remark 5: In any case, each passive coordinate is either entirely determined by the active coordinates through the kinematic model of constraints, or defined by a dynamic model. Thus, we will gather the former type of dofs into the vector $\dot{r}_{p, \text { kin }}$ of kinematic passive velocities, while the latter is gathered into $\dot{r}_{p, d y n}$, the vector of dynamic passive velocities. Formally, we will have:

$$
\dot{r}_{p, d y n}=S \dot{r}_{p}, \dot{r}_{p, k i n}=\bar{S} \dot{r}_{p},
$$

where $S$ and $\bar{S}$ are matrices of 0 and 1 which select the coordinates of dynamic and kinematic nature among the components of $\dot{r}_{p}$. In a complementary way, we will have:

$$
\dot{r}_{p}=\bar{D} \dot{r}_{p, k i n}+D \dot{r}_{p, d y n},
$$

where $D=S^{T}$ and $\bar{D}=\bar{S}^{T}$ are matrices allowing to distribute the dynamic and kinematic dofs on the vector of the passive dofs. Furthermore, since by definition the internal passive kinematic velocities are entirely specified by the internal active velocities, the general kinematic model (13) takes the particular form:

$$
\left(\begin{array}{c}
\eta \\
\dot{r}_{p}
\end{array}\right)=\left(\begin{array}{ccc}
H_{e e} & H_{e p} & J_{e x t} \\
0 & D & J_{i n t}
\end{array}\right)\left(\begin{array}{c}
\eta_{r} \\
\dot{r}_{p, d y n} \\
\dot{r}_{a}(t)
\end{array}\right) .
$$

where $J_{\text {int }}$ takes the form $J_{\text {int }}=\bar{D} \bar{J}_{\text {int }}$ which defines $\bar{J}_{\text {int }}$.

\section{Reduced dynamics of the passive dofs}

In this subsection, we consider the case 2 of remark 3 where the time integration of the passive dofs evolution requires the need of a dynamic model (i.e. $\mathcal{K}\left(A^{\ddagger}\right) \neq \varnothing$ ). This residual dynamic model corresponds to the reduced passive dynamics of the MMS controlled by the time evolution $t \mapsto r_{a}(t)$. It is derived by projecting the passive dynamics from the tangent space to $G \times \mathcal{S}_{p}$ to its admissible sub-space $\mathcal{K}\left(A^{\ddagger}\right)$. Then, timeintegrating once these dynamics will allow to compute the reduced velocities $\eta_{r}$ and $\dot{r}_{p, r}$ that can be used in a second step to reconstruct the entire motion of the MMS in space through the time-integration of the reconstruction equation (14). The projection leading to the reduced dynamics is achieved in two steps each one corresponding to the application of the reduction process (13) on the real and virtual motions, i.e. in the space of velocities and in its dual, that of forces. In this perspective, let us reconsider the reduction equation (17). It has for consequences on the (real) accelerations:

$$
\begin{aligned}
& \left(\begin{array}{c}
\dot{\eta} \\
\ddot{r}_{p}
\end{array}\right)=\left(\begin{array}{ccc}
H_{e e} & H_{e p} & J_{e x t} \\
0 & D & J_{\text {int }}
\end{array}\right)\left(\begin{array}{c}
\dot{\eta}_{r} \\
\ddot{r}_{p, d y n} \\
\ddot{r}_{a}(t)
\end{array}\right) \\
& +\left(\begin{array}{ccc}
\dot{H}_{e e} & \dot{H}_{e p} & \dot{J}_{e x t} \\
0 & 0 & \dot{J}_{\text {int }}
\end{array}\right)\left(\begin{array}{c}
\eta_{r} \\
\dot{r}_{p, d y n} \\
\dot{r}_{a}(t)
\end{array}\right) .
\end{aligned}
$$


In the same manner, we have on the virtual (dual) side the following reduction relation, which means that the field of virtual displacement used hereafter is compatible with the constraints (2):

$$
\left(\begin{array}{c}
\delta \zeta \\
\delta r_{p}
\end{array}\right)=\left(\begin{array}{cc}
H_{e e} & H_{e p} \\
0 & D
\end{array}\right)\left(\begin{array}{c}
\delta \zeta_{r} \\
\delta r_{p, d y n}
\end{array}\right)
$$

Note that equation (19) defines the vector of reduced virtual displacements (on the right), which is deduced from the virtualization of $(17)^{5}$. Now, let us consider the passive dynamics in $G \times \mathcal{S}_{p}$ controlled by the time evolution $t \mapsto r_{a}(t)$, i.e. the two first rows of (1) with $r_{a}, \dot{r}_{a}$ and $\ddot{r}_{a}$ considered as exogenous variables specified by their time-evolution. We can restate them into the form of the balance of virtual works [28], and write that for any $\left(\delta \zeta, \delta r_{p}\right)$ :

$$
\begin{gathered}
\left(\delta \zeta^{T}, \delta r_{p}^{T}\right)\left(\begin{array}{cc}
\mathcal{M} & M_{p}^{T} \\
M_{p} & m_{p p}
\end{array}\right)\left(\begin{array}{c}
\dot{\eta} \\
\ddot{r}_{p}
\end{array}\right)= \\
\left(\delta \zeta^{T}, \delta r_{p}^{T}\right)\left(\begin{array}{c}
\left(f-M_{a}^{T} \ddot{r}_{a}(t)\right)+A^{T} \lambda \\
\left(Q_{p}-m_{p a} \ddot{r}_{a}(t)\right)+B_{p}^{T} \lambda
\end{array}\right),
\end{gathered}
$$

where according to footnote $2, Q_{p}=Q_{p \text {,inert }}+Q_{p, \text { ext }}+Q_{p, \text { int }}$ and $Q_{p, i n t}$ accounts for the internal forces exerted on the passive dofs. For instance, if the $k^{t h}$ dof is a passive one introduced by a localised or a distributed compliance (on a joint or along a body respectively), the corresponding component of $Q_{p, i n t}$ is modelled by a friction and a restoring force given by (with $\mathcal{U}\left(r_{p}\right)$ a strain energy and $\mathcal{D}\left(\dot{r}_{p}\right)$ a dissipation function):

$$
Q_{p, i n t, k}=-\partial \mathcal{U} / \partial r_{p, k}-\partial \mathcal{D} / \partial \dot{r}_{p, k},
$$

while, if the $k^{t h}$ dof is introduced by an ideal passive joint, we simply have $Q_{p, i n t, k}=0$. Taking the reduction relations (18) and (19) into account allows rewriting the balance of virtual works (20) in the reduced form:

$$
\begin{gathered}
\left(\delta \zeta_{r}^{T}, \delta r_{p, d y n}^{T}\right)\left(\left(\begin{array}{cc}
\mathcal{M}_{r} & M_{p, r}^{T} \\
M_{p, r} & m_{p p, r}
\end{array}\right)\left(\begin{array}{c}
\dot{\eta}_{r} \\
\ddot{r}_{p, d y n}
\end{array}\right)\right)= \\
\left(\delta \zeta_{r}^{T}, \delta r_{p, d y n}^{T}\right)\left(\begin{array}{c}
f_{r} \\
Q_{p, r}
\end{array}\right),
\end{gathered}
$$

which represents the projection of the passive dynamics from the tangent space to $G \times \mathcal{S}_{p}$, onto the reduced subspace of admissible velocities. In (22), we introduced reduced matrices (indexed with a " $r$ ) whose detailed expressions are given in Appendix $A^{6}$. Finally, (22) being satisfied for any virtual reduced displacement, the reduced dynamics are governed by the following equations:

$$
\left(\begin{array}{cc}
\mathcal{M}_{r} & M_{p, r}^{T} \\
M_{p, r} & m_{p p, r}
\end{array}\right)\left(\begin{array}{c}
\dot{\eta}_{r} \\
\ddot{r}_{p, d y n}
\end{array}\right)=\left(\begin{array}{c}
f_{r} \\
Q_{p, r}
\end{array}\right),
$$

which once completed with the reconstruction equation (14), allow one to restate the reduced forward dynamics of passive dofs in the form of (4), (5), (7) and (8) with: $\widetilde{\mathcal{M}}_{r}=$

\footnotetext{
${ }^{5}$ Basically, such a virtualization consists in replacing $d$./ $d t$ by $\delta$. and by forcing $\delta r_{a}(t)=0$, since the actuated dofs are defined by their time evolution which is frozen along any virtual displacement [28]

${ }^{6}$ Note that due to the ideal character of contacts, the external contact forces do not appear in these expressions, since they have a null projection in the admissible space.
}

$\mathcal{M}_{r}-M_{p, r}^{T} m_{p p, r}^{-1} M_{p, r}, \widetilde{\mathcal{F}}_{r}=f_{r}-M_{p, r}^{T} m_{p p, r}^{-1} Q_{p, r}$, and $\widetilde{Q}_{p, r}=Q_{p, r}-M_{p, r} \widetilde{\mathcal{M}}_{r}^{-1} \widetilde{\mathcal{F}}_{r}$, so defining the tilde symbol.

\section{INVERSE (AND FORWARD) DYNAMICS OF THE ACTIVE DOFS}

Pursuing the resolution of the problem of section II-B, we now see that the passive motions can be entirely deduced from the time integration of (4), (5), (7) and (8). In a further step, one can use the dynamics of the MMS before the projection, i.e. (1) to calculate the external contact forces $\lambda$. To that end, let us first rewrite (1) in the "passive-active" block-partitioned form:

$$
\begin{aligned}
\left(\begin{array}{cc}
\mathcal{M}^{\ddagger} & M_{a}^{\ddagger T} \\
M_{a}^{\ddagger} & m_{a a}
\end{array}\right) & \left(\begin{array}{c}
\dot{\eta}^{\ddagger} \\
\ddot{r}_{a}(t)
\end{array}\right)= \\
& \left(\begin{array}{c}
f^{\ddagger} \\
Q_{a}+\tau_{a}
\end{array}\right)+\left(\begin{array}{c}
A^{\ddagger T} \\
B_{a}^{T}
\end{array}\right) \lambda,
\end{aligned}
$$

where $\lambda$ and $\tau_{a}$ are unknown while all the motion variables are known. Thus, one can consider the first row of (24) as an algebraic system ruling $\lambda$ along time, i.e.:

$$
\left(A^{\ddagger T}\right) \lambda+\left(\left(f^{\ddagger}-M_{a}^{\ddagger T} \ddot{r}_{a}(t)\right)-\mathcal{M}^{\ddagger} \dot{\eta}^{\ddagger}\right)=0 .
$$

The algebraic system (25) can be considered as the dual of the kinematic one (10). By generalized inversion of this system, we find:

$$
\lambda=\left(A^{\ddagger T}\right)^{(-1)}\left(\mathcal{M}^{\ddagger} \dot{\eta}^{\ddagger}-\left(f^{\ddagger}-M_{a}^{\ddagger T} \ddot{r}_{a}(t)\right)\right)+\lambda_{\text {stat }},
$$

with $\lambda_{\text {stat }} \in \mathcal{K}\left(A^{\ddagger T}\right)$. This last expression corresponds to the most general form of $\lambda$. The first term in (26) is the vector of reaction contact forces required by the motions, i.e. the part of reaction forces directly deducible from the motions. On the other hand, the second term of (26) models internal tensions which do not produce any generalized force on the passive (internal and external) dofs. Consequently, this term will not generate any passive accelerations and will be named (hyper)static reaction loading and noted $\lambda_{\text {stat }}$. Now, injecting (26) in the second row of (24) and using (11) along with $J^{T}=-\left(A^{\ddagger(-1)} B_{a}\right)^{T}$ gives:

$$
\begin{aligned}
\tau_{a} & =\left(m_{a a}+M_{a}^{\ddagger} J+J^{T} \mathcal{M}^{\ddagger} J+J^{T} M_{a}^{\ddagger T}\right) \ddot{r}_{a}(t) \\
& +\left(M_{a}^{\ddagger}+J^{T} \mathcal{M}^{\ddagger}\right)\left(H \dot{\eta}_{r}^{\ddagger}+\dot{J}_{a}(t)+\dot{H} \eta_{r}^{\ddagger}\right) \\
& -Q_{a}-J^{T} f^{\ddagger}+B_{a}^{T} \lambda_{\text {stat }} .
\end{aligned}
$$

Finally, inserting $\dot{\eta}_{r}=\widetilde{\mathcal{M}}_{r}^{-1} \widetilde{\mathcal{F}}_{r}$ into (27) gives (6) which is the general form of the internal torques exerted by the actuators onto the actuated joints. At last, removing $\lambda_{\text {stat }}$ in (27) allows one to derive the forward dynamics of actuated joints (9).

\section{A. Remarks:}

Remark 6: Going further into the details of the solutions of (25) allows to fix $\lambda_{\text {stat }}$ as given in Table I. Indeed, we will have two cases depending on the relative values of $m^{o}=\operatorname{rank}\left(A^{\ddagger T}\right)$ and $m$. When $m^{o}=m$, the system is under- or fully-constrained and $\lambda_{\text {stat }}=0$, while when $m^{o}<m$, the system is over-constrained and $\lambda_{\text {stat }} \neq 0$. 
Remark 7: When the system is over-constrained, the solutions (26) and the corresponding control torques are not univocally determined. As a result, further considerations are required to find the solutions. For instance, in a control problem, one can consider $\lambda_{\text {stat }}$ as an additional freeness that can be exploited to address other objectives than those achieved by the motion control law $t \mapsto r_{a}(t)$. In particular, depending on the friction between the bodies and the substrate, stability can be improved by controlling these internal tensions as in the case of snakes in lateral undulation [29]. From the modelling point of view, the indetermination of (26) can be removed by invoking a further model capturing the effects of other sources of compliance. As a result, these new compliances increasing the dimension of $r_{p}$, they will add new columns to $A^{\ddagger}$ and new rows to $A^{\ddagger T}$. Thus, they will increase the rank of $A^{\ddagger T}$ up to fulfill the condition $m=m^{o}$ for which the internal torques are univocally deducible from (27), with $\lambda_{\text {stat }}=0$.

\section{PRACTICAL IMPLEMENTATION OF THE APPROACH}

\section{A. Summary of the modelling approach}

Finally, in any case, the modelling approach emerges as clearly separated into several steps ordered in a two stages hierarchical approach which can be carried out in a blind manner. The first stage is of kinematic nature. It starts by stating the model of the unlocked constraints in the form of (2). Then, removing $\dot{r}_{a}$ from (2) gives the locked constraints (3). Next, applying a generalized-inversion to (3) allows deducing the kinematic model (13), which in our case, takes the particular form (17) with $J=\left(J_{\text {ext }}^{T}, J_{\text {int }}^{T}\right)^{T}$ given by (12). At this kinematic stage, the comparison of the rank of the locked and unlocked constraints (i.e. $m$ and $m^{o}$ ) also allows to predict the final form of the expected model according to Table I. In particular, if $H=0$, the modelling approach does not require any further calculation beyond the kinematic stage, while if $H \neq 0$, the kinematic model is not sufficient and the approach needs a second "dynamic" stage. The dynamic stage consists in calculating the dynamics of the system free of any constraint, i.e. (1) with $\lambda=0$. Once computed, these unconstrained (or "free") dynamics are projected according to the formulae (66-68) onto the subspace of admissible velocities $\mathcal{K}\left(A^{\ddagger}\right)$, to derive the (reduced) forward dynamics of the passive velocities (23), which once completed with the reconstruction equation (14), give the forward dynamic model of all the passive dofs. Once the forward dynamics of passive dofs are known, it just remains to compute the inverse torque dynamics with (27). Lastly, one can easily re-express the passive forward dynamics and the actuated inverse dynamics in the alternative form (4-8) while the internal actuated dynamics can be put in the forward form (9), so allowing to derive the forward dynamics of a MMS with passive internal dofs.

\section{B. Computational apects}

Returning to the locomotion problem of section II.B, we are now capable of numerically solving it through a dynamic simulation. To that end, the forward dynamics (including the reconstruction equation) of the passive dofs controlled by the motion of the actuated dofs $\left(t \mapsto\left(r_{a}, \dot{r}_{a}, \ddot{r}_{a}\right)(t)\right)$, can be computed and time-integrated once in a global time loop. Once all the motion state and acceleration variables are known at the current time, the current value of the torques supplied by the actuated joints $\tau_{a}$, can be computed before increasing the time step and to restart the algorithm. Regarding the free dynamics, when the number of bodies increases, its calculation becomes more and more complex. Consequently, the derivation of this model based on the direct calculation of the Lagrangian and the use of Lagrange-like equations as those of Poincare, quickly becomes unfeasible by hand. In order to circumvent this difficulty, we now propose an automatic and efficient computation of the free dynamics based on an extension of the recursive inverse dynamic algorithm due to Luh and Walker [24]. This well known algorithm is based on the NewtonEuler model of fully actuated Rigid Multibody Systems (RMS) [30]. Exploiting the recursive character of the Newton-Euler model, it allows to efficiently (and automatically) compute the joint torques and the reaction forces respectively exerted on the joint axes and the rigid basis of a RMS, when it is submitted to joint (shape) motions $(t \mapsto(r, \dot{r}, \ddot{r})(t))$ and rigid overall (net) motions $(t \mapsto(g, \eta, \dot{\eta})(t))$ transmitted through the mobile basis ${ }^{7}$. Going further, this algorithm is based on 2 recursions with respect to the bodies index. The first one is a forward recursion (from the basis to the manipulator tool) which computes the accelerations of the rigid bodies from the knowledge of the current basis and joints accelerations $(\dot{\eta}, \ddot{r})$ and state $(g, r, \eta, \dot{r})$. The second (backward) recursion, uses the Newton-Euler equations of the rigid bodies to compute the interbody forces from the tool to the basis. Finally, the projection of the interbody forces onto the joint axes gives the current joint torques $\tau$ while the last interbody force computed by the backward recursion is nothing but the current reaction force $f_{\text {reac }}$ exerted onto the basis. Defined as such, $\left(f_{\text {reac }}, \tau\right)$ are the external forces to be supplied in order to ensure the desired motion of the RMS. As a result, this algorithm solves the inverse dynamics of any RMS, and we will symbolically denote it as $\mathcal{D}_{R M S}^{-1}(g, r, \eta, \dot{r}, \dot{\eta}, \ddot{r})=$ $\left(f_{\text {reac }}, \tau\right)$. Since these early works, several extensions of the Luh and Walker algorithm have been proposed [15], [23], [31]. Based on the floating frame and assumed modes approach, the Luh algorithm has been extended in [23] to the case of multibody systems with compliant bodies whose all the joints are actuated. Such an extension requires to derive a generalized Newton-Euler model of Flexible Multibody Systems (FMS) detailed in [32] and reminded here in Appendix B. In this extended Luh algorithm, the elastic accelerations are outputs of the algorithm while the elastic state variables are inputs. As a result, such a recursive algorithm is symbolically denoted $\mathcal{D}_{F M S}^{-1}\left(r_{e}, g, r_{j}, \dot{r}_{e}, \eta, \dot{r}_{j}, \dot{\eta}, \ddot{r}_{j}\right)=\left(\ddot{r}_{e}, f_{\text {reac }}, \tau\right)$ with $r_{e}$ the vector of (Rayleigh-Ritz) elastic variables and $r_{j}$, that of the joint variables. Now, we are going to see how the Luh recursive algorithm can be used to calculate the free dynamics of a flexible MMS, i.e. (1) with $\lambda=0$. To that end, we first consider for the sake of pedagogy the simpler case of a RMS

\footnotetext{
${ }^{7}$ Note here that because the motion of the basis is imposed, such a system is not properly a Mobile Multibody System (MMS) in the sense that the net motions are not governed by a locomotion model.
} 
mounted on a mobile basis whose motions are imposed. With the notations introduced above, the dynamics of such a system are trivially given by:

$$
\left(\begin{array}{c}
f_{\text {reac }} \\
\tau
\end{array}\right)=\left(\begin{array}{cc}
\mathcal{M} & M^{T} \\
M & m
\end{array}\right)\left(\begin{array}{c}
\dot{\eta} \\
\ddot{r}
\end{array}\right)-\left(\begin{array}{c}
f \\
Q
\end{array}\right),
$$

which has the same form as (1) except that the passive dofs are now removed, and that the external forces transmitted by the constraints take the particular form of $\left(\lambda^{T}\left(A, B_{a}\right)\right)^{T}=$ $\left(f_{\text {reac }}^{T}, 0^{T}\right)^{T}$. Now, let us remark that in any case, $f$ and $Q$ can be written as the sum of two components, one depending on the configuration only and the other on both, the configuration and the velocities ${ }^{8}$, i.e.:

$$
\begin{aligned}
& f=f^{(p)}(g, r)+f^{(v)}(g, r, \eta, \dot{r}), \\
& Q=Q^{(p)}(g, r)+Q^{(v)}(g, r, \eta, \dot{r}) .
\end{aligned}
$$

where $Q^{(v)}(g, r, 0,0)=0$ and $f^{(v)}(g, r, 0,0)=0$. Based on this remark, all the constitutive matrices of (28) (i.e. $\mathcal{M}$, $M, m, f$ and $Q$ ) can be computed by applying $\mathcal{D}_{R M S}^{-1}$ to some sets of specific inputs. In fact, a simple examination of (28) shows that $\left(f^{(p)}, Q^{(p)}\right)=\mathcal{D}_{R M S}^{-1}(g, r, 0,0,0,0)$, $(f, Q)=-\mathcal{D}_{R M S}^{-1}(g, r, \eta, \dot{r}, 0,0)$ while if we denote $\delta_{k}$ a vector with all the components equal to zero except the $k^{t h}$ which is equal to one, we have $\left(\left(M^{T}\right)_{k}-f^{(p)},(m)_{k}-Q^{(p)}\right)=$ $\mathcal{D}_{R M S}^{-1}\left(g, r, 0,0,0, \delta_{k}\right)$ and $\left((\mathcal{M})_{k}-f^{(p)},(M)_{k}-Q^{(p)}\right)=$ $\mathcal{D}_{R M S}^{-1}\left(g, r, 0,0, \delta_{k}, 0\right)$, where $(A)_{k}$ denotes the $k^{t h}$ column of any matrix $A$. Thus, making $k$ sweep all its possible values allows to reconstruct $\mathcal{M}, M, m, f$ and $Q$ of (28). Remarkably, extending this computational process from RMS to FMS allows computing recursively the free dynamics of a MMS containing compliant bodies, at least when these bodies are terminal ones ${ }^{9}$. To show this, we first consider an equivalent FMS identical to the original MMS but with fully actuated joints and a reference body defined as a mobile basis submitted to imposed motions. The dynamic model of such a FMS is the same as that of the MMS, i.e. (1), except that the torque $\left(0^{T}, \tau_{a}^{T}\right)^{T}$ is now replaced by a full vector $\tau$ while $\lambda^{T}\left(A, B_{p}, B_{a}\right)^{T}=\left(f_{\text {reac }}^{T}, 0^{T}, 0^{T}\right)^{T}$. Moreover, separating the elastic coordinates of the bodies $r_{e}$ from those of the joints $r_{j}$ according to the block-partition $r=\left(r_{e}^{T}, r_{j}^{T}\right)$, the dynamics of such an equivalent FMS can be stated as:

$$
\left(\begin{array}{ccc}
\mathcal{M} & M_{e}^{T} & M_{j}^{T} \\
M_{e} & m_{e e} & m_{j e}^{T} \\
M_{j} & m_{j e} & m_{j j}
\end{array}\right)\left(\begin{array}{c}
\dot{\eta} \\
\ddot{r}_{e} \\
\ddot{r}_{j}
\end{array}\right)=\left(\begin{array}{c}
f \\
Q_{e} \\
Q_{j}
\end{array}\right)+\left(\begin{array}{c}
f_{\text {reac }} \\
0 \\
\tau
\end{array}\right),
$$

where let us repeat that except $\left(f_{\text {reac }}^{T}, 0^{T}, \tau^{T}\right)^{T}$, all the matrices are just an alternative block-partition of those related to the original MMS appearing in (1). As a result, if we can extend the previous recursive computational process from RMS governed by (28), to FMS governed by (29), we will be capable of recursively computing (1) with $\lambda=0$, i.e. the free

\footnotetext{
${ }^{8}$ Typically, the velocity-dependent component contains the Coriolis, centrifugal and friction forces, while the position-dependent component accounts for the restoring elastic forces and the gravity forces.

${ }^{9}$ Fortunately, this case plays an important role in compliant locomotion bio-inspired from animals which exploit the advantages of flexible terminal organs such as fish tails, insect wings and others. In the general case (of any compliant bodies) other (non-recursive) algorithms should be developed.
}

dynamics of any MMS. The point of what follows is to provide such an extension. To that end, we first reformulate the model (29) in order to conform it to the input-output map $\mathcal{D}_{F M S}^{-1}$. In particular, the elastic accelerations are some outputs of $\mathcal{D}_{F M S}^{-1}$ whose expression is given by the second row of (29):

$$
\ddot{r}_{e}=m_{e e}^{-1}\left(Q_{e}-M_{e} \dot{\eta}-m_{j e}^{T} \ddot{r}_{j}\right) .
$$

Then, re-injecting (30) into the first and third row of (29), allows to express the two other outputs of $\mathcal{D}_{F M S}^{-1}$ as:

$$
\left(\begin{array}{c}
f_{\text {reac }} \\
\tau
\end{array}\right)=\left(\begin{array}{cc}
\widetilde{\mathcal{M}} & \widetilde{M}_{j}^{T} \\
\widetilde{M}_{j} & \widetilde{m}_{j j}
\end{array}\right)\left(\begin{array}{c}
\dot{\eta} \\
\ddot{r}_{j}
\end{array}\right)-\left(\begin{array}{c}
\widetilde{f} \\
\widetilde{Q}_{j}
\end{array}\right),
$$

where we introduced the following set of matrices:

$$
\begin{aligned}
& \widetilde{\mathcal{M}}=\mathcal{M}-M_{e}^{T} m_{e e}^{-1} M_{e}, \\
& \widetilde{M}_{j}=M_{j}-m_{j e} m_{e e}^{-1} M_{e}, \\
& \widetilde{m}_{j j}=m_{j j}-m_{j e} m_{e e}^{-1} m_{j e}^{T}, \\
& \widetilde{f}=f-M_{e}^{T} m_{e e}^{-1} Q_{e}, \\
& \widetilde{Q}_{j}=Q_{j}-m_{j e} m_{e e}^{-1} Q_{e} .
\end{aligned}
$$

Now, a simple examination of (30) and (31) shows that the matrices (32-36) along with $m_{e e}^{-1} Q_{e},-m_{e e}^{-1} M_{e}$ and $-m_{e e}^{-1} m_{j e}^{T}$ appearing in (30) can be recursively computed by applying $\mathcal{D}_{F M S}^{-1}$ to specific inputs as follows (the superscripts $(p)$ and $(v)$ denote position and velocity-dependent matrices as in the rigid case): $\mathcal{D}_{F M S}^{-1}\left(r_{e}, g, r_{j}, 0,0,0,0,0,0\right)=\left(-\widetilde{f}^{(p)}, m_{e e}^{-1} Q_{e}^{(p)},-\widetilde{Q}_{j}^{(p)}\right)$, $\mathcal{D}_{F M S}^{-1}\left(r_{e}, g, r_{j}, \dot{r}_{e}, \eta, \dot{r}_{j}, 0,0,0\right)=\left(-\widetilde{f}, m_{e e}^{-1} Q_{e},-\widetilde{Q}_{j}\right)$ and $\mathcal{D}_{F M S}^{-1}\left(r_{e}, g, r_{j}, 0,0,0,0, \delta_{k}, 0\right)=\left((\widetilde{\mathcal{M}})_{k}-\right.$ $\left.\widetilde{f}^{(p)},-\left(m_{e e}^{-1} M_{e}\right)_{k}+m_{e e}^{-1} Q_{e}^{(p)}, \quad\left(\widetilde{M}_{j}\right)_{k}-\widetilde{Q}_{j}^{(p)}\right)$; $\mathcal{D}_{F M S}^{-1}\left(r_{e}, g, r_{j}, 0,0,0,0,0, \delta_{k}\right)=\left(\left(\widetilde{M}_{j}^{T}\right)_{k}-\widetilde{f}^{(p)}\right.$, $\left.-\left(m_{e e}^{-1} m_{j e}^{T}\right)_{k}+m_{e e}^{-1} Q_{e}^{(p)},\left(\widetilde{m}_{j j}\right)_{k}-\widetilde{Q}_{j}^{(p)}\right)$. Finally, once (32-36) and $\left(m_{e e}^{-1} Q_{e},-m_{e e}^{-1} M_{e},-m_{e e}^{-1} m_{j e}^{T}\right)$ known, we can infer the expressions of all the matrices of (29) by assuming the compliant bodies (if any) to be terminal bodies of the tree-like structure. In fact, in this case the computation of $m_{e e}$ is straightforward since one simply has $m_{e e}=\operatorname{diag}_{k \in \mathbb{N}_{t b}}\left(m_{k}\right)$ with $\mathbb{N}_{t b}$ the set of terminal bodies indexes ordered according to $r_{e}$, and $m_{k}$ the elastic mass matrices of the generalized Newton-Euler model (69) (see Appendix B). Then, once $m_{e e}$ is known, simple algebra allows to compute $Q_{e}, M_{e}$ and $m_{j e}^{T}$ of (30), from which we easily deduce $\mathcal{M}, f, M_{j}, m_{j j}$ and $Q_{j}$ from (32-36). Finally, we so construct all the matrices appearing in (29), except $f_{\text {reac }}$ and $\tau$ which once replaced by 0 and $\left(0^{T}, \tau_{a}^{T}\right)^{T}$ in (29) gives the original equations (1) when $\lambda=0$, i.e. the free dynamics of the original MMS. At last, returning to the numerical simulation, such calculations can be achieved numerically at each step of a global time loop or symbolically (and once for all) in order to generate with a commercial symbolic software, customized algorithms which can be executed numerically in a second step [33].

\section{APPLICATION TO WHEELED MULTIBODY SYSTEMS}

We now consider two examples of wheeled systems chosen for their illustrative value. The first example belongs to the 


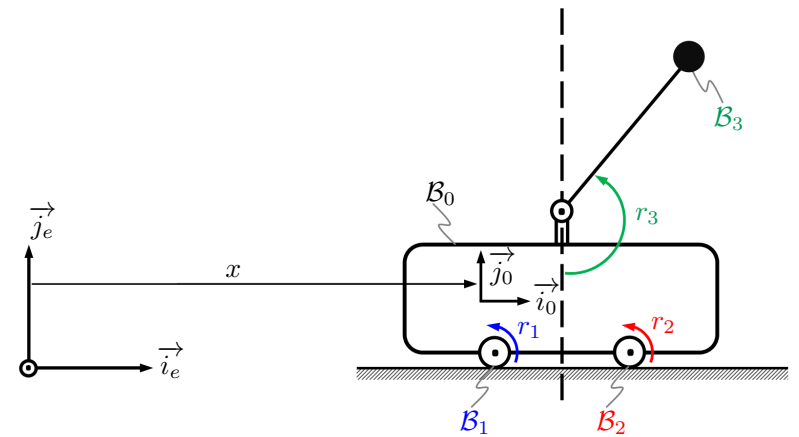

Fig. 2. Frames and parametrization of a mobile pendulum

class of systems containing passive internal joints. It is a mobile pendulum with one internal passive dof coupled to a wheeled cart supported by two redundant wheels. The second case belongs to the class of systems whose passive internal dofs are purely kinematic. This is the $3 \mathrm{D}$ bicycle, a very familiar system but whose dynamics are rarely exhibited in their full generality.

\section{A. The mobile pendulum}

This is a planar cart supported by two wheels constrained to roll without slipping along a one-dimensional rail (see Fig. 2 ). On the cart, a single pendulum of length $l$ and mass $m$, is pinned through a single dof angular joint. All the joints and contacts are assumed to be ideal (no friction, no elasticity). All the mass of the pendulum is concentrated at its tip. The two identical wheels have a mass $m_{w}$ and an inertia momentum (about their center) $J_{w}$. This system has four bodies: the two wheels $\mathcal{B}_{1}$ and $\mathcal{B}_{2}$, the cart considered as the reference body $\mathcal{B}_{0}$ and the pendulum $\mathcal{B}_{3}$. In this trivial case where the constraints are holonomic, the space of configurations $\mathbb{R} \times \mathcal{S}$ is directly compatible with the constraints. Thus the reduction process is quite artificial. Nevertheless, for the sake of illustration we are going to apply the general framework developed in section III to this system when both wheels are active. This example allows to illustrate the previous general context in the overconstrained case where the control torques are not unique. The group of net displacements is identified here to the one dimensional translations $x \in \mathbb{R}$, where $x$ denotes the position of the cart along the rail. As regards the shape space, we split it into $\mathcal{S}=\mathcal{S}_{p} \times \mathcal{S}_{a}$ with coordinates $r_{p}=r_{3}$ and $r_{a}=\left(r_{1}, r_{2}\right)^{T}$. The constraints are imposed by the rolling without slipping conditions of the two wheels along the rail, i.e.:

$$
\left(\begin{array}{ll}
1 & 0 \\
1 & 0
\end{array}\right)\left(\begin{array}{c}
\dot{x} \\
\dot{r}_{3}
\end{array}\right)-\left(\begin{array}{cc}
R & 0 \\
0 & R
\end{array}\right)\left(\begin{array}{l}
\dot{r}_{1} \\
\dot{r}_{2}
\end{array}\right)=\left(\begin{array}{l}
0 \\
0
\end{array}\right),
$$

which defines $A^{\ddagger}$ and $B_{a}$ of $(10,68)$ and shows that $m=$ $n+s_{p}=2$ and $m^{o}=1$. As a result, we are in the case where $n+s_{p}>m^{o}$ and $m>m^{o}$, and from Table I, we know that $H \neq 0$ and $\lambda_{\text {stat }} \neq 0$. Now, a generalized inversion of (37) gives:

$$
\left(\begin{array}{c}
\dot{x} \\
\dot{r}_{3}
\end{array}\right)=\left(\begin{array}{cc}
R & 0 \\
0 & 0
\end{array}\right)\left(\begin{array}{c}
\dot{r}_{1} \\
\dot{r}_{2}
\end{array}\right)+\left(\begin{array}{c}
0 \\
1
\end{array}\right) \dot{r}_{3}
$$

which defines $J=-A^{\ddagger(-1)} B_{a}$, with $\mathcal{K}\left(A^{\ddagger}\right)$ spanned by $H=$ $(0,1)^{T}$. Furthermore, identifying (38) with the general case (17) allows to define the two nonzero matrices $J_{\text {ext }}=(R, 0)$ and $D=1$. Then, we have to compute the free dynamics of this system in the space $\mathbb{R} \times \mathcal{S}_{p} \times \mathcal{S}_{a}$, and find (with $\gamma=$ $9.81 \mathrm{Nm}^{2}$ the gravity acceleration, and $M$ the total mass of the system (cart, pendulum and two wheels)):

$$
\left(\begin{array}{cccc}
M & m l c 3 & 0 & 0 \\
m l c 3 & m l^{2} & 0 & 0 \\
0 & 0 & J_{w} & 0 \\
0 & 0 & 0 & J_{w}
\end{array}\right)\left(\begin{array}{c}
\ddot{x} \\
\ddot{r}_{3} \\
\ddot{r}_{1} \\
\ddot{r}_{2}
\end{array}\right)=\left(\begin{array}{c}
m l s 3 \dot{r}_{3}^{2} \\
-m l s 3 \gamma \\
\tau_{1} \\
\tau_{2}
\end{array}\right),
$$

which is the particular form of (1) (with $\lambda=0$ ) for the mobile pendulum. Now applying the general reduction process (66) and (67), with the only nonzero matrices: $\mathcal{M}=M, M_{p}=$ $m l c 3, m_{p p}=m l^{2}, f_{\text {inert }}=m l s 3 \dot{r}_{3}^{2}, Q_{p, e x t}=-m l s 3 \gamma$ and $m_{a a}=J_{w} 1_{2}$, gives:

$$
m l^{2} \ddot{r}_{3}=-m l \gamma s 3-m l R c 3 \ddot{r}_{1}(t),
$$

that has to be completed with the reconstruction equation:

$$
\dot{x}=R \dot{r_{1}} .
$$

Then, in order to compute the internal torque dynamics (27), we also need:

$$
A^{\ddagger T}=\left(\begin{array}{ll}
1 & 1 \\
0 & 0
\end{array}\right),
$$

whose kernel is defined by:

$$
\mathcal{K}\left(A^{\ddagger T}\right)=\left\{\left(\begin{array}{c}
+1 \\
-1
\end{array}\right) T / T \in \mathbb{R}\right\},
$$

that gives after straightforward computations:

$$
\begin{gathered}
\left(\begin{array}{c}
\tau_{1} \\
\tau_{2}
\end{array}\right)=\left(\begin{array}{cc}
J_{w}+M R^{2} & 0 \\
0 & J_{w}
\end{array}\right)\left(\begin{array}{l}
\ddot{r}_{1} \\
\ddot{r}_{2}
\end{array}\right) \\
+\left(\begin{array}{c}
m R l\left(c 3 \ddot{r}_{3}-s 3 \dot{r}_{3}^{2}\right) \\
0
\end{array}\right)+\left(\begin{array}{cc}
R & 0 \\
0 & R
\end{array}\right)\left(\begin{array}{c}
+1 \\
-1
\end{array}\right) T,
\end{gathered}
$$

which is true for any $T$. Finally, the expression of the wheels' control torque (27) appears as the sum of a component supplying the external force required by the motion, and another which models eventual antagonist internal torques producing no motion. Note that due to our choice of generalized inversion, taking $T=0$ makes the front wheel the leading one. On the contrary, taking $T=-\left(M R^{2} \ddot{r}_{1}+m R l\left(c 3 \ddot{r}_{3}-s 3 \dot{r}_{3}^{2}\right)\right) / R$ shifts the external force on the rear wheel, while taking $T=-\left(M R^{2} \ddot{r}_{1}+m R l\left(c 3 \ddot{r}_{3}-s 3 \dot{r}_{3}^{2}\right)\right) / 2 R$ distributes the forces equally between the two wheels. Note that this last case could be directly obtained by taking the pseudo-inverse as a particular generalized inverse.

\section{B. The bicycle}

The bicycle is modelled by a MMS with four connected rigid bodies as drawn on Fig. 3, where its frame defines the reference body $\mathcal{B}_{0}$ of the MMS. The bicycle moves on a planar ground without sliding nor slipping, however, it can tilt. Since we aim at modelling a 3D locomotion system, the configuration space is $S E(3) \times \mathcal{S}$ with the shape space here defined by $S^{1} \times S^{1} \times S^{1}$ which stands for the dofs of the 


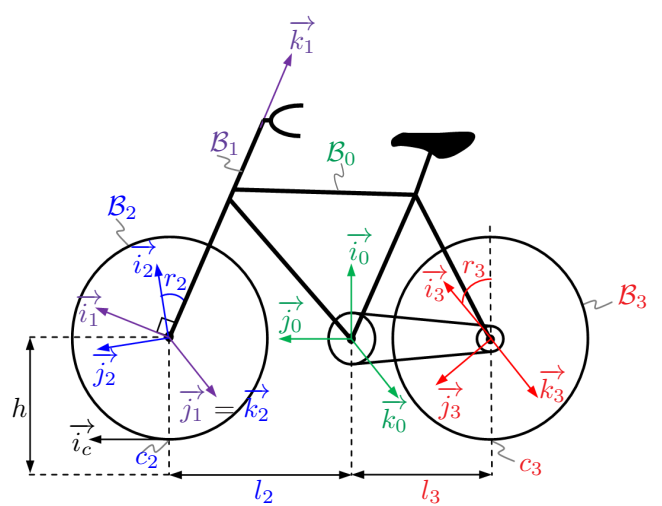

Fig. 3. Frames and parametrization of a bicycle

handlebars and the two wheels parameterized by the angles $r_{1}, r_{2}$ and $r_{3}$ respectively. The rear wheel is directly actuated by the two pedals which exert a torque $\tau_{3}$ on it. The handlebars are also actuated by $\tau_{1}$ while the front wheel is passive but constrained by the motions of the other dofs. As a result, we split $\mathcal{S}$ into $\mathcal{S}=\mathcal{S}_{p} \times \mathcal{S}_{a}$ with $S_{p}$ and $\mathcal{S}_{a}$ coordinatized by $r_{p}=r_{2}$ and $r_{a}=\left(r_{1}, r_{3}\right)$ respectively. As regards the geometric definition of the bicycle, the frames of the bodies and the design parameters are indicated on Fig. 3. We assume that the wheels are identical and modelled by two planar discs of mass $m_{w}$ and of inertia momentum $I_{w}$ and $J_{w}$, depending on whether the corresponding axis, which passes through their mass centers $\mathrm{O}_{2}$ and $\mathrm{O}_{3}$, is coplanar or perpendicular to the wheel, respectively. On the other hand, the bicycle frame is a $3 \mathrm{D}$ rigid body defined by its mass $m_{0}$, the vector of its first momenta in the reference frame $\left(m X_{0}, m Y_{0}, m Z_{0}\right)^{T}$ and the matrix of its second momenta:

$$
\left(\begin{array}{ccc}
X X_{0} & X Y_{0} & X Z_{0} \\
Y X_{0} & Y Y_{0} & Y Z_{0} \\
Z X_{0} & Z Y_{0} & Z Z_{0}
\end{array}\right)
$$

which is nothing more than the angular inertia matrix of $\mathcal{B}_{0}$ in its reference frame. Finally, we neglect the inertia of the bike's fork and handlebars around the steering axis, with respect to that introduced by the front wheel.

1) Kinematic constraints: We assume that contacts between the wheels and the ground are perfect (no friction, no deformation). As a result, the constraints reflect the fact that in any direction normal to their plane, each wheel cannot penetrate nor separate from the ground at the contact point, while in a direction parallel to their plane, the wheels roll without slipping. Then, it remains to invoke the parametrization of the velocities imposed by our definition of the configuration space (as fiber bundle), to obtain the six constraints in the form of (2) with:

$$
A=\left(\begin{array}{cccccc}
1 & 0 & 0 & 0 & 0 & 0 \\
0 & 1 & 0 & 0 & 0 & 0 \\
0 & 0 & 1 & -l_{3} & 0 & 0 \\
0 & -c 1 & -c \alpha s 1 & -l_{2} c \alpha s 1 & 0 & 0 \\
-s \alpha s 1 & -c \alpha s 1 & c 1 & l_{2} c_{1} & 0 & l_{2} s \alpha c 1 \\
0 & 0 & 0 & 0 & 0 & 1
\end{array}\right),
$$

$$
B_{p}=\left(\begin{array}{c}
0 \\
0 \\
0 \\
h \\
0 \\
0
\end{array}\right), B_{a}=\left(\begin{array}{cc}
0 & 0 \\
0 & -h \\
0 & 0 \\
h s \alpha c \alpha s 1 & 0 \\
0 & 0 \\
0 & 0
\end{array}\right) .
$$

A simple analysis of these constraints shows that $m^{o}=$ $\operatorname{rank}\left(A, B_{p}\right)=6=m$. Then, since $n+s_{p}=6+1=7$, we are in the case where: $n+s_{p}>m^{o}, m=m^{o}$. Hence, from Table I, we have $H \neq 0$ and $\lambda_{\text {stat }}=0$.

2) Kinematic model: Now, it is possible to use a generalized inversion of $A^{\ddagger}=\left(A, B_{p}\right)$ to derive the kinematic model (13). However, we will derive the model in a more straightforward way as follows. First, let us remark that from the first and last row of the constraints (46-47) we find:

$$
V_{0 X}=0, \Omega_{0 Z}=0 .
$$

Then, injecting (48) in the third and fifth row of (46-47) gives:

$$
V_{0 Z}=\left(\frac{l_{3} c \alpha \tan 1}{l_{2}+l_{3}}\right) V_{0 Y}, \Omega_{0 X}=\left(\frac{c \alpha \tan 1}{l_{2}+l_{3}}\right) V_{0 Y},
$$

while $\Omega_{0 Y}$ is undetermined, and as such, defines the kernel of $A^{\ddagger}$. Now, let us remark that all the other net velocities are defined by $V_{0 Y}$ and that $V_{0 Y}$ is defined from $\dot{r}_{3}$ using the second row of (46-47) through the relation:

$$
V_{0 Y}=h \dot{r}_{3} .
$$

Finally, all the components of the net velocities are fixed by $\dot{r}_{3}$ except the tilt velocity $\Omega_{0 Y}$ which requires a dynamic model to be determined. This fact is confirmed by the following relation:

$$
\left(\begin{array}{c}
V_{0 X} \\
V_{0 Y} \\
V_{0 Z} \\
\Omega_{0 X} \\
\Omega_{0 Y} \\
\Omega_{0 Z}
\end{array}\right)=\left(\begin{array}{c}
0 \\
h \\
\psi l_{3} \\
\psi \\
0 \\
0
\end{array}\right) \dot{r}_{3}+\left(\begin{array}{l}
0 \\
0 \\
0 \\
0 \\
1 \\
0
\end{array}\right) \Omega_{0 Y} .
$$

with:

$$
\psi\left(r_{1}\right)=\frac{h c \alpha \tan 1}{l_{2}+l_{3}} .
$$

On the other hand, if we inject the constraints (48), (49) and (51) in the fourth row of (46-47), we find:

$$
\dot{r}_{2}=\left(c 1+c^{2} \alpha s 1 \tan 1\right) \dot{r}_{3}-s \alpha c \alpha s 1 \dot{r}_{1},
$$

This last constraint (53) allows one to deduce the motion of the front free wheel from that of the rear wheel and the handlebars, both being actuated. Finally, gathering (51) and (53) with $r_{a}=$ $\left(r_{1}, r_{3}\right)^{T}$ and $r_{p}=r_{p, \text { kin }}=r_{2}$, gives the model (17), with $D=0$ and $H_{e p}=0$ (since all the passive dofs are kinematic) and the following expressions:

$$
\begin{aligned}
J_{\text {int }}^{T}= & \bar{J}_{\text {int }}^{T}=\left(\begin{array}{c}
-s \alpha c \alpha s 1 \\
c 1+c^{2} \alpha s 1 \tan 1
\end{array}\right), \\
J_{\text {ext }}= & \left(\begin{array}{cc}
0 & 0 \\
0 & h \\
0 & \psi l_{3} \\
0 & \psi \\
0 & 0 \\
0 & 0
\end{array}\right), H_{e e}=\left(\begin{array}{l}
0 \\
0 \\
0 \\
0 \\
1 \\
0
\end{array}\right) .
\end{aligned}
$$


In the next subsection, the kinematic model (54) is used to reduce the bicycle dynamics.

3) Reduced passive dynamics: As suggested before, we first derive the free dynamics of the bicycle by applying the previous recursive algorithm in its rigid version, which gives the model in the form of (1) with $\lambda=0$. In the case of the bicycle, the expressions of the matrices appearing in this model are given in Appendix C. In a second step, one applies the reduction process (66-68), to obtain the reduced dynamics equation (23) which here becomes:

$$
\mathcal{M}_{r} \dot{\Omega}_{0 Y}=f_{r},
$$

with:

$$
\begin{gathered}
\mathcal{M}_{r}=H_{e e}^{T} \mathcal{M} H_{e e}, \\
f_{r}=H_{e e}^{T}\left(f-\mathcal{M} \dot{J}_{e x t} \dot{r}_{a}-M_{p}^{T} \dot{J}_{i n t} \dot{r}_{a}\right) .
\end{gathered}
$$

Hereby let us remind that $f=f_{\text {ext }}+f_{\text {inert }}, Q_{p}=Q_{p \text {,ext }}+$ $Q_{p, \text { inert }}$ and $Q_{a}=Q_{a, \text { ext }}+Q_{a, \text { inert }}$, while if the rear wheel has no friction $Q_{p, i n t}=0$. Finally, the reduced dynamics have to be completed with the kinematic model of net motions:

$$
\dot{g}=g\left(H_{e e} \Omega_{0 Y}+J_{e x t}\left(\begin{array}{c}
\dot{r}_{1} \\
\dot{r}_{3}
\end{array}\right)\right),
$$

which once gathered with (53) forms the two reconstruction equations (7-8) for the bicycle. In all these expressions, the matrices appearing in the free dynamics are given in Appendix C, while $H_{e e}, J_{\text {int }}$ and $J_{\text {ext }}$ are detailed in (54). Finally, introducing the same data into the general expression of the control torques (27) with $\lambda_{\text {stat }}=0$, gives the two control torques $\tau_{a}=\left(\tau_{1}, \tau_{3}\right)^{T}$.

\section{APPLICATION TO SOFT ROBOTICS}

The third example illustrates a locomotion system mixing non-holonomic constraints and soft robotics. This system is obtained by reconsidering the snake-board of [1] where the rigid actuated rotor accumulating the kinetic momentum (which is cyclically transferred to the external dynamics through the constraints), is now replaced by two soft appendages symmetrically positioned with respect to the motor shaft (Fig. 4). This system could be used to explore the potential benefits of cyclically storing and restoring the kinetic energy in the compliant rotor.

\section{A. The soft snake-board}

For the sake of simplicity, the wheels are not declared in the multibody structure but only taken into account through their kinematic model while their mass is added to that of the platform. With this choice, the snake-board is a MMS with five bodies. The platform $\mathcal{B}_{0}$, the two axles $\mathcal{B}_{1}$ and $\mathcal{B}_{2}$ and finally the flexible rotor that we model by two symmetric flexible bodies $\mathcal{B}_{3}$ and $\mathcal{B}_{4}$. These two bodies are rigidly cantilevered on a vertical shaft actuated by a motor attached to the platform in the origins of the beam frames $O_{3}=O_{4}$. The configuration space is $S E(2) \times \mathcal{S}$ with $\mathcal{S}=\mathcal{S}_{p} \times \mathcal{S}_{a}$ and the coordinates $r=\left(r_{p}^{T}, r_{a}^{T}\right)^{T}$. As in [1] we impose that the angles of the two axles with the platforms are opposite, so that the vector of active coordinates can be reduced to $r_{a}=\left(r_{1}, r_{3}\right)^{T}$, with $r_{1}$ and $r_{2}=-r_{1}$ the angles of the two axles, and $r_{3}$ that of the rotor, all measured with respect to the platform (Fig. 4). The remaining coordinates are defined by $r_{p}=r_{p, d y n}=r_{e}$ with $r_{e}$ the vector of elastic (modal) coordinates of the flexible bodies. To fix the ideas, $\mathcal{B}_{3}$ and $\mathcal{B}_{4}$ will be modelled as two identical Euler-Bernoulli planar beams undergoing flexural deformations described in the basis of the cantilever modes of the two beams [20]. We will take one mode per beam so that $r_{e}=\left(r_{e 3}, r_{e 4}\right)$ where the first coordinate is that of the first mode of $\mathcal{B}_{3}$ and the second, that of $\mathcal{B}_{4}$, i.e.:

$$
d_{3}\left(X_{3}\right)=\phi_{1}\left(X_{3}\right) r_{e 3}, d_{4}\left(X_{4}\right)=\phi_{1}\left(X_{4}\right) r_{e 4},
$$

with $d_{3} \vec{j}_{3}$ and $d_{4} \vec{j}_{4}$ the two fields of transverse deformation along the two beam axis $\left(O_{3}, X_{3}\right)$ and $\left(O_{4}, X_{4}\right)$ respectively (Fig. 4) and $\phi_{1}$ the first flexural mode of the beam. With all these definitions, stating that the wheels roll on a planar ground without slipping nor sliding allowing to write the constraints under the general form (2) with $m=m^{o}=2$ and $n+s_{p}=$ $3+2=5$. Thus from Table $\mathrm{I}$, we have $H \neq 0$ and $\lambda_{\text {stat }}=$ 0 . Then, simple handling of these constraints allows one to derive the kinematic model of the elastic snake-board under the general form (17) which becomes in this case:

$$
\left(\begin{array}{c}
V_{0 X} \\
V_{0 Y} \\
\Omega_{0 Z} \\
\dot{r}_{e 3} \\
\dot{r}_{e 4}
\end{array}\right)=\left(\begin{array}{ccccc}
-2 l \cos ^{2}\left(r_{1}\right) & 0 & 0 & 0 & 0 \\
0 & 0 & 0 & 0 & 0 \\
\sin \left(2 r_{1}\right) & 0 & 0 & 0 & 0 \\
0 & 1 & 0 & 0 & 0 \\
0 & 0 & 1 & 0 & 0
\end{array}\right)\left(\begin{array}{c}
\eta_{r} \\
\dot{r}_{e 3} \\
\dot{r}_{e 4} \\
\dot{r}_{1}(t) \\
\dot{r}_{3}(t)
\end{array}\right),
$$

Now, in order to compute the external unconstrained dynamics of the system which in this case take the form of (29) with $f_{\text {reac }}=0$ and $\lambda=0$, one can use the recursive algorithm proposed in section V.B. Remarkably, since there are no more than two bodies (including $\mathcal{B}_{0}$ ) per branches, the outputs of $\mathcal{D}_{F M S}^{-1}$ (i.e. $\left.\left(f_{\text {reac }}, \ddot{r}_{e}, \tau\right)\right)$ can be computed explicitly by hand as follows (we use the notations of the generalized NewtonEuler model reminded in Appendix B):

$$
f_{\text {reac }}=\widetilde{\mathcal{M}} \dot{\eta}+\widetilde{M}_{j}^{T} \ddot{r}_{j}-\widetilde{f},
$$

with:

$$
\begin{aligned}
& \widetilde{\mathcal{M}}=\mathcal{M}_{0}+ \\
& \sum_{k=1}^{2} A d_{g_{k, 0}}^{T} \mathcal{M}_{k} A d_{g_{k, 0}}+\sum_{k=3}^{4} A d_{g_{k, 0}}^{T} \widetilde{\mathcal{M}}_{k}^{+} A d_{g_{k, 0}}
\end{aligned}
$$

and:

$$
\begin{aligned}
& M_{j}^{T} \ddot{r}_{j}-\tilde{f}=\mathcal{F}_{0}+ \\
& \sum_{k=1}^{2} A d_{g_{k, 0}}^{T}\left(-\mathcal{M}_{k} \zeta_{k}+\mathcal{F}_{k}\right)+\sum_{k=3}^{4} A d_{g_{k, 0}}^{T}\left(-\widetilde{\mathcal{M}}_{k}^{+} \zeta_{k}+\widetilde{\mathcal{F}}_{k}^{+}\right)
\end{aligned}
$$
with $\widetilde{\mathcal{M}}_{k}^{+}=\mathcal{M}_{k}-M_{k}^{T} m_{k}^{-1} M_{k}$ and $\widetilde{\mathcal{F}}_{k}^{+}=\mathcal{F}_{k}-M_{k}^{T} m_{k}^{-1} Q_{k}$. In the same manner, the elastic accelerations can be detailed as (for $k=3,4)$ :

$$
\ddot{r}_{e k}=m_{k}^{-1}\left(-M_{k}\left(A d_{g_{k, 0}} \dot{\eta}+\zeta_{k}\right)+Q_{k}\right),
$$

while, the internal torques are given by:

$$
\begin{array}{r}
\tau_{k}=A_{k}^{T}\left[\left(\mathcal{M}_{k}-M_{k}^{T} m_{k}^{-1} M_{k}\right)\left(A d_{g_{k, 0}} \dot{\eta}+\zeta_{k}\right)\right. \\
\left.-\mathcal{F}_{k}+M_{k}^{T} m_{k}^{-1} Q_{k}\right] .
\end{array}
$$




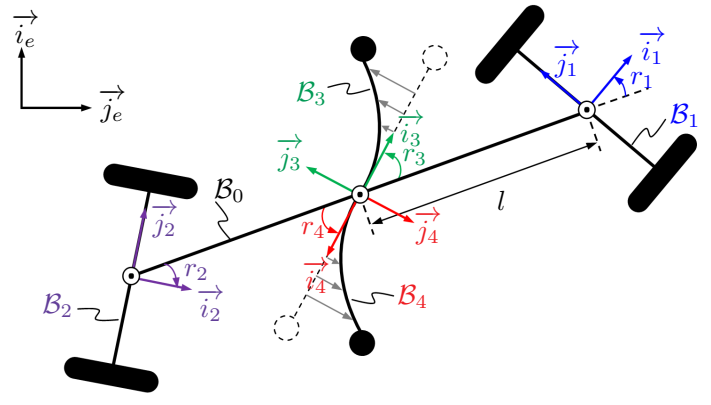

Fig. 4. Frames and parametrization of an elastic snake-board

Then, according to the algorithm of section V.B, forcing $\ddot{r}_{j}=$ 0 in (63) gives $\widetilde{f}$, while forcing $\left(\dot{r}_{j}, \eta\right)=(0,0)$ and taking specific joint accelerations, allows to construct $\widetilde{M}_{j}^{T}$ column by column. In the same manner, imposing specific inputs in the expression of torques (65) and elastic accelerations (64) allows one to compute $\widetilde{m}_{j j}$ and $\widetilde{Q}_{j}$, along with $m_{e e}^{-1} Q_{e}$, $-m_{e e}^{-1} M_{e}$ and $-m_{e e}^{-1} m_{j e}^{T}$. Finally, since $m_{e e}=m_{f 1} 1_{2}$ with $m_{f 1}$ the modal mass of the first flexural mode of one of the two identical appendages and $1_{2}$ the $2 \times 2$ identity matrix, we can recover all the matrices of (29). Then projecting these dynamics onto the admissible space using the reduction formula (66-68) with $H_{e e}=\left(-2 l \cos ^{2}\left(r_{1}\right), 0, \sin \left(2 r_{1}\right)\right)^{T}$, $H_{e p}=0_{3 \times 2}, J_{e x t}=0_{3 \times 2}, J_{i n t}=0_{2 \times 2}$ and $D=1_{2}$, finally gives after a few rearrangements, the dynamics in the form of (4-8). At last, using (27) with the same matrices and $\lambda_{\text {stat }}=0$, gives the three expected control torques.

\section{CONCLUSION}

This article contributes to propose a general formulation for the locomotion dynamics of any mobile multibody system containing passive internal degrees of freedom and subject to kinematic constraints and/or external forces. The final set of equations includes the forward dynamics of the passive (external and internal) degrees of freedom (or "passive dynamics") along with the (inverse and forward) dynamics of the actuated joints. The implementation of the approach is systematic and structured in two stages: a kinematic and a dynamic stage. The kinematic stage starts with the computation of the set of kinematic constraints, from which a kinematic model is derived through a general inversion procedure. In the most general case, this kinematic model mixes two contributions. The first one is a pure kinematic contribution for which the corresponding passive (internal and external) velocities are entirely deducible from the actuated internal ones. The second contribution is governed by a dynamic model deduced from the projection of the unconstrained dynamics of the MMS onto the kernel of the constraints while the actuated joints are locked. The final formulation can cope with a wide range of systems including MMS with passive (free or compliant) joints ruled by dynamics (as in the case of compliant walkers and pendular climbers) or free joints ruled by kinematics (as in the case of non-holonomic wheeled MMS). Finally, it can also model MMS which contain bodies with distributed compliances, as this is the case of bio-inspired robots exploiting the virtues of soft propulsive appendages. More than giving the final form of the equations, the approach also gives access to a classification of the systems depending on the relative values of some intrinsic numbers as the dimensions of the passive dofs and the rank of the locked and unlocked independent constraints. Finally, the article also proposes a simple algorithm to compute the unconstrained dynamics of these MMS (that have to be projected after). This algorithm is in itself new, and based on an extension of the Luh's recursive algorithm from rigid to flexible multibody systems. At the end, the approach is applied to several systems chosen for their illustrative interest. In future, this general set of equations will be used to study more advanced cases of bio-inspired soft locomotion such as that inspired by the hovering flight of moths or the swimming of fish with undulating tail. Finally, we also intend to program this algorithm using Mathematica to generate automatically customized (optimized) symbolic models.

\section{APPENDIX A}

EXPRESSIONS OF THE REDUCED MATRICES OF (23)

The reduced inertia matrix can be detailed as:

$$
\begin{aligned}
& \left(\begin{array}{cc}
\mathcal{M}_{r} & M_{p, r}^{T} \\
M_{p, r} & m_{p p, r}
\end{array}\right)= \\
& \left(\begin{array}{cc}
H_{e e}^{T} & 0 \\
H_{e p}^{T} & D^{T}
\end{array}\right)\left(\begin{array}{ll}
\mathcal{M} & M_{p}^{T} \\
M_{p} & m_{p p}
\end{array}\right)\left(\begin{array}{cc}
H_{e e} & H_{e p} \\
0 & D
\end{array}\right),
\end{aligned}
$$

while the reduced forces are given by:

$$
\left(\begin{array}{c}
f_{r} \\
Q_{p, r}
\end{array}\right)=\left(\begin{array}{cc}
H_{e e}^{T} & 0 \\
H_{e p}^{T} & D^{T}
\end{array}\right)\left(\begin{array}{c}
F \\
Q
\end{array}\right),
$$

with:

$$
\begin{aligned}
& \left(\begin{array}{c}
F \\
Q
\end{array}\right)=-\left(\begin{array}{cc}
\mathcal{M} & M_{p}^{T} \\
M_{p} & m_{p p}
\end{array}\right) \\
& \left(\begin{array}{c}
\dot{H}_{e e} \eta_{r}+\dot{H}_{e p} \dot{r}_{p, d y n}+\dot{J}_{e x t} \dot{r}_{a}(t)+J_{e x t} \ddot{r}_{a}(t) \\
\dot{J}_{i n t} \dot{r}_{a}(t)+J_{i n t} \ddot{r}_{a}(t)
\end{array}\right)+ \\
& +\left(\begin{array}{c}
f-M_{a}^{T} \ddot{r}_{a}(t) \\
Q_{p}-m_{p a} \ddot{r}_{a}(t)
\end{array}\right) .
\end{aligned}
$$

\section{APPENDIX B}

GENERALIZED NEWTON-EULER MODEL OF FMS

Here, we remind the Generalized Newton-Euler model of a MMS containing compliant bodies. Following usual NewtonEuler conventions on tree-like structures labeling [34], the indices of bodies increase from the reference body $\mathcal{B}_{0}$ to the terminal organs as instantiated in Fig. 1, while the indices $i$ and $l$ are reserved to denote the antecedent and the successor of the current index $k$, respectively. According to [32], in the case where the assumed modes used to describe the deformation of each of the bodies are cantilevered, such a model can be stated as follows:

- Generalized Newton-Euler model of the bodies:

$$
\begin{gathered}
\left(\begin{array}{cc}
\mathcal{M}_{k} & M_{k}^{T} \\
M_{k} & m_{k}
\end{array}\right)\left(\begin{array}{c}
\dot{\eta}_{k} \\
\ddot{r}_{e k}
\end{array}\right)= \\
\left(\begin{array}{c}
\mathcal{F}_{k} \\
Q_{k}
\end{array}\right)+\left(\begin{array}{c}
F_{k}-\sum_{l / k=a(l)} A d_{g_{l, k}}^{T} F_{l} \\
-\sum_{l / k=a(l)}^{/ k} \Phi_{k}^{T} R_{g_{k, l}} F_{l}
\end{array}\right) .
\end{gathered}
$$


- Kinematic model of the transformations:

$$
g_{k}=g_{i} g_{e i} g_{r i} \text {. }
$$

- Kinematic model of the velocities:

$$
\eta_{k}=A d_{g_{k, i}} \eta_{i}+R_{g_{k, i}} \Phi_{i} \dot{r}_{e i}+A_{k} \dot{r}_{k} .
$$

- Kinematic model of the accelerations:

$$
\dot{\eta}_{k}=A d_{g_{k, i}} \dot{\eta}_{i}+R_{g_{k, i}} \Phi_{i} \ddot{r}_{e i}+\zeta_{k} .
$$

As any Newton-Euler model, this model is structured in two sets of equations. The first (69) corresponds to the dynamic balance of isolated bodies ${ }^{10}$, the second (70-72) models the kinematic constraints imposed by the joints ${ }^{11}$. In (69-72), $r_{k}$ is the $k^{t h}$ joint coordinate, $r_{e k}$ is the vector of the modal coordinates of the $k^{t h}$ body, $g_{k}$ is the transformation mapping a unique frame fixed to space onto the $k^{\text {th }}$ body (floating) frame, $\eta_{k}=g_{k}^{-1} \dot{g}_{k}$ is the corresponding $(6 \times 1)$ velocity vector (in the mobile frame), $F_{k}$ is the inter-body $(6 \times 1)$ force vector exerted by $\mathcal{B}_{i}$ onto $\mathcal{B}_{k}, A d_{g_{k, i}}$ (respect. $R_{g_{k, i}}$ ) is the matrix changing a velocity from the $i^{t h}$ floating frame (respect. basis) to its successor. The transformation $g_{e k}$ represents the (elastic) transformation introduced by the deformation of $\mathcal{B}_{k}$ in its connection point with its successor, while $\Phi_{k} \dot{r}_{e k}=\dot{g}_{e k} g_{e k}^{-1}$ is the corresponding $(6 \times 1)$ (elastic) velocity vector. The transformation $g_{r k}$ represents the transformation that would map the $i^{t h}$ floating frame onto its successor, if the MMS was rigid (this is the rigid geometric model of the MMS). Finally, the corresponding velocity is $A_{k} \dot{r}_{k}=g_{r k}^{-1} \dot{g}_{r k}$ which accounts for the joint velocity while $\zeta_{k}$ contains the corresponding accelerations $\left(\ddot{r}_{k}\right)$ as well as all the residual velocity dependent accelerations resulting from the time-differentiation of (71). The reader interested in the detailed expression of these quantities is referred to [32]. Finally, since $\mathcal{B}_{0}$ is the reference body which defines the external motions, one has: $\left(g_{0}, \eta_{0}, \dot{\eta}_{0}\right)=(g, \eta, \dot{\eta})$.

\section{APPENDIX C}

FREE DYNAMICS OF THE BICYCLE

The matrices appearing in the bicycle's free dynamics can be obtained using the recursive algorithm proposed in section V. They take the form of (1), with $\lambda=0$ and:

$$
\mathcal{M}=\left(\begin{array}{cc}
m 1 & m \widehat{s}^{T} \\
m \widehat{s} & I
\end{array}\right) \text {. }
$$

Whose computation, requires the expressions:

$$
\begin{gathered}
m=2 m_{w}+m_{o}, \\
m \widehat{s}^{T}= \\
\left(\begin{array}{ccc} 
& \\
0 & 0 & m_{w}\left(l_{3}-l_{2}\right)-m Y_{0} \\
-m Z_{0} & 0 & m X_{0} \\
m_{w}\left(l_{2}-l_{3}\right)+m Y_{0} & -m X_{0} & 0
\end{array}\right),
\end{gathered}
$$

\footnotetext{
${ }^{10}$ In (69), one finds from left to right the Generalized Newton-Euler inertia matrix, the vector of body-reference and modal accelerations, the vector of generalized inertia, restoring, and external forces (except the inter-body ones), and finally the vector of inter-body generalized forces.

${ }^{11}$ Equation (70) (for which (71) and (72) are differential consequences) just models how one can shift from one floating frame to its successor along the structure.
}

$$
I=\left(\begin{array}{ccc}
I_{x x} & I_{x y} & I_{x z} \\
I_{y x} & I_{y y} & I_{y z} \\
I_{z x} & I_{z y} & I_{z z}
\end{array}\right)
$$

where:

$$
\begin{aligned}
I_{x x} & =X X_{0}+m_{w}\left(l_{2}^{2}+l_{3}^{2}\right)+I_{w}+X X \\
I_{x y} & =I_{y x}=X Y+X Y_{0} \\
I_{x z} & =I_{z x}=X Z+X Z_{0} \\
I_{y y} & =Y Y_{0}+I_{w}+Y Y \\
I_{y z} & =I_{z y}=Y Z+Y Z_{0} \\
I_{z z} & =Z Z+J_{w}+Z Z_{0}+m_{w}\left(l_{2}^{2}+l_{3}^{2}\right),
\end{aligned}
$$

with:

$$
\begin{aligned}
& X X=s \alpha^{2}\left(I_{w} c 1^{2}+J_{w} s_{1}^{2}\right)+I_{w} c \alpha^{2} \\
& X Y=Y X=s \alpha c \alpha\left(I_{w} c 1^{2}+J_{w} s 1^{2}-I_{w}\right) \\
& X Z=Z X=\left(I_{w}-J_{w}\right) s \alpha s 1 c 1 \\
& Y Y=c \alpha^{2}\left(I_{w} c 1^{2}+J_{w} s 1^{2}\right)+I_{w} s \alpha^{2} \\
& Y Z=Z Y=\left(I_{w}-J_{w}\right) c \alpha s 1 c 1 \\
& Z Z=I_{w} s_{1}^{2}+J_{w} c_{1}^{2},
\end{aligned}
$$

while:

$$
\begin{aligned}
M_{p}^{T}= & \left(\begin{array}{c}
0 \\
0 \\
0 \\
-s \alpha s 1 J_{w} \\
-c \alpha s 1 J_{w} \\
J_{w} c 1
\end{array}\right), M_{a}^{T}=\left(\begin{array}{cc}
0 & 0 \\
0 & 0 \\
0 & 0 \\
c \alpha I_{w} & 0 \\
-s \alpha I_{w} & 0 \\
0 & J_{w}
\end{array}\right) \\
& \left(\begin{array}{cc}
m_{p p} & m_{p a} \\
m_{a p} & m_{a a}
\end{array}\right)=\left(\begin{array}{ccc}
J_{w} & 0 & 0 \\
0 & I_{w} & 0 \\
0 & 0 & J_{w}
\end{array}\right)
\end{aligned}
$$

In the following expressions we also use the "linear-angular" block-partition:

$$
\left(\begin{array}{cc}
M_{p}^{T} & M_{a}^{T}
\end{array}\right)=\left(\begin{array}{c}
M_{l i n} \\
M_{a n g}
\end{array}\right) .
$$

As far as the right hand side of (1) is concerned, we have:

$$
f_{\text {inert }}=\left(\begin{array}{c}
f_{\text {inert }, \text { lin }} \\
f_{\text {inert }, \text { ang }}
\end{array}\right) \text {, }
$$

with:

$$
\begin{aligned}
f_{\text {inert }, \text { lin }} & =\Omega_{0} \times m V_{0}+\Omega_{0} \times\left(\Omega_{0} \times m s\right) \\
f_{\text {inert,ang }} & =m s \times\left(\Omega_{0} \times V_{0}\right)+\Omega_{0} \times\left(I \Omega_{0}+M_{a n g} \dot{r}\right) \\
& +\frac{\partial I}{\partial r_{1}} \Omega_{0} \dot{r}_{1}-J_{w}\left(\begin{array}{c}
s \alpha c 1 \\
c \alpha c 1 \\
s 1
\end{array}\right) \dot{r}_{1} \dot{r}_{2}
\end{aligned}
$$

whereas:

$$
\begin{gathered}
Q_{a, \text { inert }}=\frac{1}{2}\left(\begin{array}{c}
\Omega_{0}^{T} \frac{\partial I}{\partial r_{1}} \Omega_{0} \\
0
\end{array}\right)+ \\
+\left(\begin{array}{c}
-J_{w}\left(s \alpha c 1 \Omega_{0 X}+c \alpha c 1 \Omega_{0 Y}+s 1 \Omega_{0 Z}\right) \dot{r}_{1} \\
0
\end{array}\right), \\
Q_{p, \text { inert }}=J_{w} \dot{r}_{1}\left(s \alpha c 1 \Omega_{0 X}+c \alpha c 1 \Omega_{0 Y}+s 1 \Omega_{0 Z}\right),
\end{gathered}
$$

while $Q_{p, \text { int }}$ can model the friction introduced by the rear wheel's joint. Finally, since the external forces are induced 
by the gravity only, they can directly be deduced from the previous context, by replacing $\dot{V}_{0}$ by the acceleration field of the earth expressed in the reference frame of the bicycle, i.e.: $R^{T} \Upsilon$, with $\Upsilon \simeq(0,0, \gamma)^{T}$. This gives:

$$
\begin{aligned}
& f_{\text {ext }}=\left(\begin{array}{cc}
m 1 & m \widehat{s}^{T} \\
m \widehat{s} & I
\end{array}\right)\left(\begin{array}{c}
R^{T} \Upsilon \\
0
\end{array}\right)=\left(\begin{array}{c}
m R^{T} \Upsilon \\
m s \times R^{T} \Upsilon
\end{array}\right), \\
& \left(\begin{array}{c}
Q_{p, \text { ext }} \\
Q_{a, \text { ext }}
\end{array}\right)=\left(\begin{array}{c}
M_{p} \\
M_{a}
\end{array}\right)\left(\begin{array}{c}
R^{T} \Upsilon \\
0
\end{array}\right)=\left(\begin{array}{l}
0 \\
0
\end{array}\right) .
\end{aligned}
$$

\section{REFERENCES}

[1] J. Ostrowski, J. W. Burdick, A. D. Lewis, and R. M. Murray, "The mechanics of undulatory locomotion: The mixed kinematic and dynamic case," in Proc. IEEE Int. Conf. Robot. Autom., 1995, pp. 1945-1951.

[2] S. D. Kelly and R. M. Murray, "Geometric phases and robotic locomotion," J. Robotic Systems, vol. 12, no. 6, pp. 417-431, 1995.

[3] J. W. Burdick, J. Radford, and G. S. Chirikjian, "A 'sidewinding' locomotion gait for hyper-redundant robots," in Proc. IEEE Int. Conf. Robot. Autom., May. 1993, pp. 101-106.

[4] R. M. Alexander, Principles of Animal Locomotion. Princeton University Press, 2002.

[5] R. Pfeifer, M. Lungarella, and F. Iida, "Self-organization, embodiment, and biologically inspired robotics," Science, vol. 318, no. 5853, pp. 1088-1093, 2007.

[6] T. J. Roberts and E. Azizi, "Flexible mechanisms: The diverse roles of biological springs in vertebrate movement," Journal of Experimental Biology, vol. 214, no. 3, pp. 353-361, 2011.

[7] K. Schmidt-Nielsen, "Locomotion: Energy cost of swimming, flying, and running," Science, vol. 177, no. 4045, pp. 222-228, 1972.

[8] J. Bertram, A. Ruina, C. Cannon, Y. Chang, and M. Coleman, "A pointmass model of gibbon locomotion," Journal of Experimental Biology, vol. 202, no. 19, pp. 2609-2617, 1999.

[9] S. I. Jensen-Segal, S. Virost, and W. R. Provancher, "ROCR: Dynamic vertical wall climbing with a pendular two-link mass-shifting robot," IEEE International Conference on Robotics and Automation, 2008. ICRA 2008, pp. 3040-3045, 2008.

[10] W. R. Provancher, S. I. Jensen-Segal, and M. A. Fehlberg, "ROCR: An energy-efficient dynamic wall-climbing robot," IEEE/ASME Transactions on Mechatronics, vol. 16, no. 5, pp. 897-906, 2011.

[11] J. C. Liao, "A review of fish swimming mechanics and behaviour in altered flows," Philosophical Transactions of the Royal Society of London - Series B: Biological Sciences, vol. 362, no. 1487, pp. 19731993, 2007.

[12] F. Candelier, M. Porez, and F. Boyer, "Note on the swimming of an elongated body in a non-uniform flow," Journal of Fluid Mechanics, vol. 716, pp. 616-637, 2013.

[13] E. A. Shammas, H. Choset, and A. A. Rizzi, "Towards a unified approach to motion planning for dynamic underactuated mechanical systems with non-holonomic constraints," The International Journal of Robotics Research, vol. 26, no. 10, pp. 1075-1124, 2007.

[14] J. D. G. Kooijman, J. P. Meijaard, J. M. Papadopoulos, A. Ruina, and A. L. Schwab, "A bicycle can be self-stable without gyroscopic or caster effects," Science, vol. 332, no. 6027, pp. 339-342, 2011.

[15] F. Boyer and S. Ali, "Recursive inverse dynamics of mobile multibody systems with joints and wheels," IEEE Transactions on Robotics, vol. 27, no. 2, pp. $215-228$, April 2011.

[16] J. P. Ostrowski and J. W. Burdick, "The geometric mechanics of undulatory robotics locomotion," The International Journal of Robotics Research, vol. 17, no. 7, pp. 683-701, 1998.

[17] J. Ostrowski, "Computing reduced equations for robotic systems with constraints and symmetries," IEEE Transactions on Robotics and Automation, vol. 15, no. 1, pp. 111-123, Feb 1999.

[18] A. M. Bloch, P. S. Krishnaprasad, J. E. Marsden, and R. M. Murray, "Nonholonomic mechanical systems with symmetry," Archive for Rational Mechanics and Analysis, vol. 136, no. 1, pp. 21-99, 1996.

[19] J. Canavin and P. Likins, "Floating reference frames for flexible spacecraft," Journal of Spacecraft and Rockets, vol. 14, no. 12, pp. 924-732, 1977.

[20] L. Meirovitch, Dynamics and Control of structures. Wiley, New York, 1989.

[21] P. C. Hughes and G. B. Sincarsin, "Dynamics of elastic multibody chains: Part B-Global dynamics," Dynamics and Stability of Systems, vol. 4, no. 3-4, pp. 227-243, 1989.
[22] C. Damaren and I. Sharf, "Simulation of flexible-link manipulators with inertial and geometric nonlinearities," Journal of Dynamic Systems, Measurement and Control, vol. 117, no. 1, pp. 74-87, 1995.

[23] F. Boyer and W. Khalil, "An efficient calculation of flexible manipulator inverse dynamics," International Journal of Robotic Research, vol. 17, no. 3, pp. 282-293, 1998.

[24] M. W. Walker, J. Y. S. Luh, and R. C. P. Paul, "On-line computational scheme for mechanical manipulator," Transaction ASME, J. of Dyn. Syst., Measurement and Control, vol. 102, no. 2, pp. 69-76, 1980.

[25] H. Poincaré, "Sur une forme nouvelle des équations de la mécanique," Comptes Rendus de l'Académie des Sciences de Paris, vol. 132, pp. 369-371, 1901.

[26] N. Chetaev, "Sur les équations de Poincaré," Comptes-rendus de l'Académie des Sciences de Paris, vol. 185, pp. 1577-1578, 1927.

[27] J. Park and W.-K. Chung, "Geometric integration on euclidean group with application to articulated multibody systems," IEEE Transactions on Robotics, vol. 21, no. 5, pp. 850-863, Oct. 2005.

[28] H. Goldstein, Classical Mechanics, 3rd ed. Addison Wesley, 2001

[29] F. Boyer, S. Ali, and M. Porez, "Macro-continuous dynamics for hyperredundant robots: Application to kinematic locomotion bio-inspired by elongated body animals," IEEE Transactions on Robotics, vol. 28, no. 2, pp. 303 - 317, April 2012.

[30] R. M. Murray, S. S. Sastry, and L. Zexiang, A Mathematical Introduction to Robotic Manipulation, 1st ed. Boca Raton, FL, USA: CRC Press, Inc., 1994.

[31] W. Khalil, G. Gallot, and F. Boyer, "Dynamic modeling and simulation of a 3-D serial eel like robot," IEEE Trans on Sys, Man, and Cybernetics, Part C: Applications and Reviews, vol. 37, no. 6, pp. 1259-1268, 2007.

[32] F. Boyer and P. Coiffet, "Generalization of Newton-Euler model for flexible manipulators," Journal of Robotic Systems, vol. 13, no. 1, pp. $11-24,1996$.

[33] P. Khosla, "Real-time control and identification of direct drive manipulators," Ph.D. dissertation, Carnegie Mellon University, Pittsburgh, PA, 1986.

[34] W. Khalil and J.-F. Kleinfinger, "Minimum operations and minimum parameters of the dynamic models of tree structure robots," IEEE Journal of Robotics and Automation, vol. 3, no. 6, pp. 517-526, December 1987.

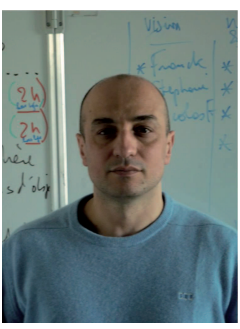

Frédéric Boyer was born in France in 1967. He received a Diploma in Mechanical Engineering from the Institut Nationale Polytechnique de Grenoble, Grenoble, France in 1991, a Master of Research degree in Mechanics from the University of Grenoble in 1991 and a Ph.D. degree in Robotics from the University of Paris VI, Paris, France in 1994. He is currently a Professor at the Department of Automatic Control, Ecole des Mines de Nantes, Nantes, France where he works with the Robotics Team, Institut de Recherche en Communication et Cybernétique de Nantes (IRCCyN). His current research interests include structural dynamics, geometric mechanics, and bio-robotics. Prof. Boyer was awarded the Monpetit prize of the Academy of Science of Paris in 2007 for the whole of his work in Dynamics.

Ayman Belkhiri received a Licence degree in Physics from Louis Pasteur University (ULP), Strasbourg, France in 2007 and a Master of Research degree in Fluid Mechanics from Pierre and Marie Curie University (UPMC), Paris, France in 2009. He is currently working toward a Ph.D. degree at the Ecole des Mines de Nantes at the Institut de Recherche en Communication et Cybernétique de Nantes (IRCCyN) on the dynamic modeling of deformable wings for MAVs bio-inspired by insects. 